\title{
Higher rank Clifford indices of curves on a K3 surface
}

\author{
Soheyla Feyzbakhsh ${ }^{1} \cdot$ Chunyi $_{\mathrm{Li}^{2}}$
}

Accepted: 19 April 2021 / Published online: 14 June 2021

(C) The Author(s) 2021

\begin{abstract}
Let $(X, H)$ be a polarized $\mathrm{K} 3$ surface with $\operatorname{Pic}(X)=\mathbb{Z} H$, and let $C \in|H|$ be a smooth curve of genus $g$. We give an upper bound on the dimension of global sections of a semistable vector bundle on $C$. This allows us to compute the higher rank Clifford indices of $C$ with high genus. In particular, when $g \geq r^{2} \geq 4$, the rank $r$ Clifford index of $C$ can be computed by the restriction of Lazarsfeld-Mukai bundles on $X$ corresponding to line bundles on the curve $C$. This is a generalization of the result by Green and Lazarsfeld for curves on K3 surfaces to higher rank vector bundles. We also apply the same method to the projective plane and show that the rank $r$ Clifford index of a degree $d(\geq 5)$ smooth plane curve is $d-4$, which is the same as the Clifford index of the curve.
\end{abstract}

Keywords Clifford index $\cdot$ Bridgeland stability conditions $\cdot$ K3 surface

Mathematics Subject Classification 14F05 $\cdot 14 \mathrm{H} 50 \cdot 14 \mathrm{~J} 28$

\section{Contents}

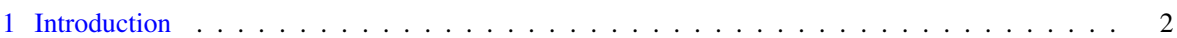

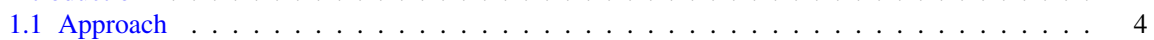

2 Review: stability conditions, wall-crossings . . . . . . . . . . . . . . . . . . 4

3 Bounds for the dimension of global sections . . . . . . . . . . . . . . . . . 8

3.1 The destabilizing wall for a stable vector bundle on the curve $C \ldots \ldots \ldots$. . . . . . 8

3.2 An upper bound on the dimension of global sections . . . . . . . . . . . . . . . . . . . 12

4 Higher rank Clifford indices . . . . . . . . . . . . . . . . . . . . . . . . . . . . . 19

$\bowtie$ Chunyi Li

C.Li.25@warwick.ac.uk

https://sites.google.com/site/chunyili0401/

Soheyla Feyzbakhsh

s.feyzbakhsh@imperial.ac.uk

https://www.imperial.ac.uk/people/s.feyzbakhsh

1 Mathematics Department, Imperial College, Huxley Building, London SW7 2AZ, UK

2 Mathematics Institute, University of Warwick, Coventry CV4 7AL, UK

Birkhäuser 
4.1 Picard number one case . . . . . . . . . . . . . . . . . . . . . . . . . . . 19

4.2 Higher Picard number case . . . . . . . . . . . . . . . . . . . . . 27

5 Smooth plane curves . . . . . . . . . . . . . . . . . . . . . . . . 27

5.1 Review: space of geometric stability conditions on $D^{b}\left(\mathbf{P}^{2}\right) \ldots \ldots \ldots \ldots \ldots \ldots$

5.2 Upper bound on the dimension of global sections $\ldots \ldots \ldots \ldots \ldots \ldots$

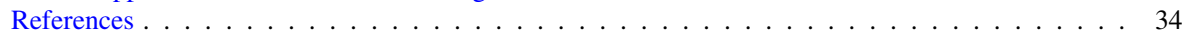

\section{Introduction}

Let $\mathfrak{U}_{C}(r, d)$ be the set of semistable rank $r$-vector bundles of degree $d$ on a smooth curve $C$. For $E \in \mathfrak{U}_{C}(r, d)$, its Clifford index is defined as

$$
\operatorname{Cliff}(E)=\frac{d}{r}-\frac{2}{r} h^{0}(C, E)+2 .
$$

By the higher rank Clifford Theorem [3, Theorem 2.1], when $0 \leq d \leq r(g-1)$, the index Cliff $(E)$ is non-negative. The rank $r$ Clifford index of $C$, first introduce in [15] where it was denoted $\gamma_{r}^{\prime}$, is defined as:

$$
\operatorname{Cliff}_{r}(C):=\min \left\{\operatorname{Cliff}(E) \mid E \in \mathfrak{U}_{C}(r, d), d \leq r(g-1), h^{0}(C, E) \geq 2 r\right\} .
$$

Our main result is as follows.

Theorem 1.1 Let $(X, H)$ be a smooth polarized K3 surface satisfying Assumption(*), and let $C$ be a smooth curve of genus $g$ in the linear system $|H|$. Let $E$ be a slope semistable rank $r$-vector bundle of degree $d$ on the curve $C$ such that $d \leq r(g-1)$. Then we have the bound for the dimension of the global sections of $E$ :

$$
h^{0}(C, E)<r+\frac{g}{4 r(g-1)^{2}} d^{2}+\frac{r}{g} .
$$

When $r \geq 2$ and $g \geq r^{2}$, the rank $r$ Clifford index of $C$

$$
\operatorname{Cliff}_{r}(C)=\frac{2}{r}(g-1)-\frac{2}{r}\left\lfloor\frac{g}{r}\right\rfloor .
$$

Assumption (*) $H^{2}$ divides $H \cdot D$ for all curve classes $D$ on $X{ }^{1}$

The upper bound for $h^{0}(C, E)$ in Theorem 1.1 is much stronger than the higher rank Clifford Theorem, which says $h^{0}(C, E) \leq r+\frac{d}{2}$. The bound is not far from the sharp bound, see Remark 3.5. For a smooth curve $C$ of genus g, several upper bounds for the dimension of global sections of vector bundles of low slope $\mu=d / r$ have been introduced in $[3,22,23]$, which are also included in [17]. Sharp bounds for the case

\footnotetext{
1 In particular, $X$ satisfies Assumption $(*)$ if $\operatorname{Pic}(X)=\mathbb{Z} H$. By the surjectivity of the period map, there are polarized K3 surfaces with higher Picard rank satisfying this assumption as well. To simplify the presentation, we explain our entire argument in the case of Picard rank one and then explain in Sect. 4.2 why the argument works for all polarized K3 surfaces satisfying Assumption (*).
} 
$g \leq 6$ and $\mu<2$ have been determined in [3,17-19,22,23]. The upper bound (1) is in general stronger than the bounds in these previous papers unless $g \leq 6$ or $\mu \leq 2$.

For $r=2$, the second statement of Theorem 1.1 gives

$$
\operatorname{Cliff}_{2}(C)=\operatorname{Cliff}(C)=\left\lfloor\frac{g-1}{2}\right\rfloor,
$$

so we re-obtain the result [1, Theorem 1.3]. Also for $r \geq 3$ and $g \neq 10$, we have

$$
\operatorname{Cliff}_{r}(C)<\operatorname{Cliff}(C)=\left\lfloor\frac{g-1}{2}\right\rfloor .
$$

This indicates the failure of the Mercat's conjecture in [24] for $C$ which states the higher ranks Clifford indices of the curve $C$ are equal to Cliff $(C)$. Meanwhile, when $r=3$ and $g=10$, we have $\operatorname{Cliff}_{3}(C)=4=\operatorname{Cliff}(C)$ for a general curve.

When $r=3$ and $g=9$, the fact that a general curve has $\operatorname{Cliff}_{3}(C)=\frac{10}{3}$ was known according to the results in [16]. When $r=3$ and $g=11$, our result implies that a general curve has $\mathrm{Cliff}_{3}(C)=\frac{14}{3}$, which improves the result $\frac{11}{3} \leq \mathrm{Cliff}_{3}(C) \leq \frac{14}{3}$ in [14, Theorem 3.6].

Let $A$ be a globally generated line bundle on the curve $C \subset X$, the LazarsfeldMukai bundle $E_{C, A}$ on $X$ is defined via the exact sequence

$$
0 \rightarrow E_{C, A}^{\vee} \rightarrow H^{0}(C, A) \otimes \mathcal{O}_{X} \stackrel{\text { ev }}{\rightarrow} A \rightarrow 0
$$

In all cases in the second part of Theorem 1.1, there exists a line bundle $A$ on the curve $C$ such that the rank $r$-Clifford index is computed by the restriction of the corresponding Lazarsfeld-Mukai bundle on the K3 surface $X$. We expect this result holds without the assumption on the Picard group of $X$. This can be viewed as a generalization for the result of Green and Lazarsfeld [10] which says that for a curve $C$ on a smooth K3 surface with Cliff $(C)<\left\lfloor\frac{g-1}{2}\right\rfloor$, the Clifford index can be computed by the restriction of a line bundle on the $\mathrm{K} 3$ surface.

Our argument can be generalized to curves on other surfaces, especially when the surface admits a stronger Bogomolov-Gieseker type inequality. Examples of such surfaces include the projective plane, del Pezzo surfaces and quintic surfaces. We explain more details for smooth plane curves in Sect. 5. In particular, we show that the first part of the Mercat's conjecture [24] holds for smooth plane curves:

Theorem 1.2 (Corollary 5.6) Let $C$ be a degree l $(\geq 5)$ smooth irreducible plane curve, then

$$
\operatorname{Cliff}_{r}(C)=l-4,
$$

for any positive integer $r$.

The result $\mathrm{Cliff}_{2}(C)=l-4$ for plane curves first appeared in [15, Proposition 8.1]. Further discussions for the rank 3 case appeared in [16]. In particular, the result 
$\mathrm{Cliff}_{3}(C)=l-4$ was known for $l \leq 6$. As Professor Peter Newstead pointed out, it seems to us that all other Clifford indices for smooth plane curves have not been known. In particular, Theorem 1.2 excludes the possibility that $\mathrm{Cliff}_{3}(C)<l-4$ in the assumption in [16, Theorem 5.6].

Another concrete example for curves on degree four del Pezzo surfaces is computed in [13]. The Clifford type inequality for such curves is the key ingredient in proving the existence of Bridgeland stability conditions on smooth quintic threefolds.

\subsection{Approach}

The main tool in this paper is the notion of stability condition introduced by Bridgeland [4]. In general, such a stability condition $\sigma=(\mathcal{A}, Z)$ is defined on a $\mathbb{C}$-linear triangulated category $T$, and is consisting of a heart structure $\mathcal{A}$ and a central charge $Z: K(T) \rightarrow \mathbb{C}$, which is a group homomorphism from the Grothendieck group to complex numbers. The space of stability conditions on $T$ forms a complex manifold which admits a wall and chamber decomposition for any fixed object $E \in T$. In this paper, the triangulated category $T$ will always be the bounded derived category $D^{b}(X)$ of coherent sheaves on a surface $X$. We will only make use of a real two-dimensional subspace of stability conditions on $D^{b}(X)$.

Let $\iota: C \hookrightarrow X$ be the embedding of a smooth curve $C$ into the surface $X$, and let $E$ be a semistable vector bundle on the curve $C$. In [8], a new upper bound for the dimension of global sections of objects in $D^{b}(X)$ has been introduced. This states the dimension of global sections of $\iota_{*} E$ can be bounded by the length of the HarderNarasimhan polygon at a limit point $\sigma_{0}$ where $Z\left(\mathcal{O}_{X}\right) \rightarrow 0$. The Harder-Narasimhan polygon geometrically represents the slopes and degrees of the Harder-Narasimhan factors of $\iota_{*} E$ with respect to $\sigma_{0}$. One of the key parts of the paper is to describe the position of the wall for $\iota_{*} E$ that bounds the large volume limit chamber at where $\iota_{*} E$ is stable. Describing the wall that bounds the large volume limit will enable us to control the length of this Harder-Narasimhan polygon at $\sigma_{0}$ effectively and get the bound for the dimension of global sections of the vector bundle $E$.

\section{Review: stability conditions, wall-crossings}

Let $(X, H)$ be a smooth polarized $\mathrm{K} 3$ surface over $\mathbb{C}$ with $\operatorname{Pic}(X)=\mathbb{Z} H$. In this section, we review the description of a slice of the space of stability conditions $\operatorname{Stab}(X)$ on $D^{b}(X)$ given in [5, Section 1-7].

Given an object $E \in D^{b}(X)$, we write $\operatorname{ch}(E)=\left(\operatorname{rk}(E), \operatorname{ch}_{1}(E), \operatorname{ch}_{2}(E)\right) \in$ $H^{*}(X, \mathbb{Z})$ for its Chern characters. We write $H_{\mathrm{alg}}^{*}(X, \mathbb{Z})$ for its algebraic part, in other words, the image of $\operatorname{ch}(-)$. The slope of a coherent sheaf $E \in \operatorname{Coh} X$ is defined by

$$
\mu_{H}(E):= \begin{cases}\frac{H \cdot \operatorname{ch}_{1}(E)}{H^{2} \operatorname{rk}(E)} & \text { if } \operatorname{rk}(E)>0 \\ +\infty & \text { if } \operatorname{rk}(E)=0\end{cases}
$$

This leads to the usual notion of $\mu_{H}$-stability. For any $\beta \in \mathbb{R}$, we have the following torsion pair in $\operatorname{Coh} X$ 


$$
\begin{aligned}
& \mathcal{T}^{\beta}:=\left\langle E: E \text { is } \mu_{H} \text {-semistable with } \mu_{H}(E)>\beta\right\rangle, \\
& \mathcal{F}^{\beta}:=\left\langle E: E \text { is } \mu_{H} \text {-semistable with } \mu_{H}(E) \leq \beta\right\rangle
\end{aligned}
$$

where $\langle-\rangle$ denotes the extension-closure. Following [5,11], this lets us define a new heart of a bounded t-structure in $\mathrm{D}^{b}(X)$ as follows:

$$
\begin{aligned}
& \operatorname{Coh}^{\beta} X:=\left\langle\mathcal{F}^{\beta}[1], \mathcal{T}^{\beta}\right\rangle \\
& \quad=\left\{E: \mathrm{H}^{-1}(E) \in \mathcal{F}^{\beta}, \mathrm{H}^{0}(E) \in \mathcal{T}^{\beta}, \mathrm{H}^{i}(E)=0 \text { for } i \neq 0,-1\right\} .
\end{aligned}
$$

For any pair $(\beta, \alpha) \in \mathbb{R}^{2}$, we define the central charge $Z_{\beta, \alpha}: K(X) \rightarrow \mathbb{C}$ by

$$
Z_{\beta, \alpha}(E):=-\operatorname{ch}_{2}(E)+\alpha \operatorname{rk}(E)+i\left(\frac{H \operatorname{ch}_{1}(E)}{H^{2}}-\beta \operatorname{rk}(E)\right) .
$$

Note that the function $Z_{\beta, \alpha}$, up to the action of $\mathrm{GL}^{+}(2 ; \mathbb{R})$, is the same as the stability function defined in [5, section 6]. The function $Z_{\beta, \alpha}$ factors via the Chern character

$$
\operatorname{ch}: K(X) \rightarrow H_{\text {alg }}^{*}(X, \mathbb{Z}) \cong \mathbb{Z}^{3}, \quad \operatorname{ch}(E)=\left(\operatorname{rk}(E), \operatorname{ch}_{1}(E), \operatorname{ch}_{2}(E)\right)
$$

The kernel of $Z_{\beta, \alpha}$ in $H_{\mathrm{alg}}^{*}(X, \mathbb{R})$ under the basis $\left\{\mathrm{rk}, \mathrm{ch}_{1}, \mathrm{ch}_{2}\right\}$ is spanned by $(1, \beta H, \alpha)$.

Definition 2.1 Let $\gamma: \mathbb{R} \rightarrow \mathbb{R}$ be a 1-periodic function such that for $x \in\left[-\frac{1}{2}, \frac{1}{2}\right]$ is defined as

$$
\gamma(x):= \begin{cases}\left(1-x^{2}\right) & \text { if } x \neq 0 \\ 0 & \text { if } x=0\end{cases}
$$

Let $\Gamma(x):=\frac{H^{2}}{2} x^{2}-\gamma(x)$. By abuse of notations, we also denote the graph of $\Gamma$ by curve $\Gamma$ (see Fig. 1).

We first state Bridgeland's result describing stability conditions on $\mathrm{D}^{b}(X)$, and then expand upon the statements.

Theorem 2.2 [5, Section 1] For any pair $(\beta, \alpha) \in \mathbb{R}^{2}$ such that $\alpha>\Gamma(\beta)$, the pair $\sigma_{\beta, \alpha}:=\left(\operatorname{Coh}^{\beta} X, Z_{\beta, \alpha}\right)$ defines a stability condition on $D^{b}(X)$. Moreover, the map from $\Gamma_{+}:=\{(\beta, \alpha) \in \mathbb{R} \times \mathbb{R} \mid \alpha>\Gamma(\beta)\} \rightarrow \operatorname{Stab}(X)$ is continuous.

We first explain the notion of $\sigma_{\beta, \alpha}$-stability and the associated Harder-Narasimhan filtration. Consider the slope function

$$
v_{\beta, \alpha}: \quad \operatorname{Coh}^{\beta} X \rightarrow \mathbb{R} \cup\{+\infty\}, \quad v_{\beta, \alpha}(E):= \begin{cases}-\frac{\Re Z_{\beta, \alpha}(E)}{\Im Z_{\beta, \alpha}(E)} & \text { if } \Im Z_{\beta, \alpha}(E)>0 \\ +\infty & \text { if } \Im Z_{\beta, \alpha}(E)=0 .\end{cases}
$$

This defines a notion of stability in $\operatorname{Coh}^{\beta} X$ : an object $E \in \operatorname{Coh}^{\beta} X$ is $\sigma_{\beta, \alpha}$-(semi)stable if and only if it is (semi)stable with respect to the slope function $v_{\beta, \alpha}$. Every object 
Fig. 1 The Gamma curve

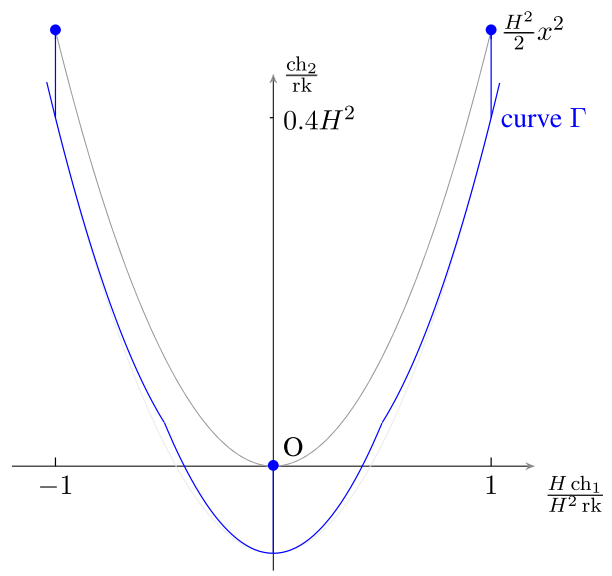

$E \in \mathrm{Coh}^{\beta} X$ admits a Harder-Narasimhan filtration which is a finite sequence of objects in $\operatorname{Coh}^{\beta} X$,

$$
0=F_{0} \subset F_{1} \subset F_{2} \subset \cdots \subset F_{k}=E
$$

whose factors $E_{i}:=F_{i} / F_{i-1}$ are $\sigma_{\beta, \alpha}$-semistable and $v_{\beta, \alpha}\left(E_{1}\right)>v_{\beta, \alpha}\left(E_{2}\right)>\cdots>$ $v_{\beta, \alpha}\left(E_{k}\right)$. We denote $v_{\beta, \alpha}^{+}(E):=v_{\beta, \alpha}\left(E_{1}\right)$ and $v_{\beta, \alpha}^{-}(E):=v_{\beta, \alpha}\left(E_{k}\right)$. The second part of Theorem 2.2 implies that the two-dimensional family of stability conditions $\sigma_{\beta, \alpha}$ satisfies wall-crossing as $\alpha$ and $\beta$ vary. Consider the projection

$$
p r: H_{\mathrm{alg}}^{*}(X, \mathbb{Z}) \backslash\{\mathrm{rk}=0\} \rightarrow \mathbb{R}^{2}, \quad \operatorname{pr}(\operatorname{ch}(E))=\left(\frac{H \operatorname{ch}_{1}(E)}{H^{2} \operatorname{rk}(E)}, \frac{\operatorname{ch}_{2}(E)}{\operatorname{rk}(E)}\right)
$$

By abuse of notations, we use the same plane for the image of the projection $p r$ and the $(\beta, \alpha)$-plane. Note that the point $(\beta, \alpha)$ is equal to the projection $\operatorname{pr}\left(\operatorname{ker} Z_{\beta, \alpha}\right)$ of the kernel of the central charge $Z_{\beta, \alpha}$ in $H_{\mathrm{alg}}^{*}(X, \mathbb{Z})$. We will also write $\operatorname{pr}(E)$ instead of $\operatorname{pr}(\operatorname{ch}(E))$.

Remark 2.3 (a) For a stable object $E$ with respect to any stability condition $\sigma_{\beta, \alpha}$, the point $\operatorname{pr}(E)$ is not in $\Gamma_{+}=\left\{(x, y) \in \mathbb{R}^{2}: y>\Gamma(x)\right\}$. To see this, note that

$$
\begin{aligned}
2 & =\operatorname{hom}(E, E)+\operatorname{hom}(E, E[2]) \geq \chi(E, E) \\
& =2 \operatorname{rk}(E) \operatorname{ch}_{2}(E)-\left(\operatorname{ch}_{1}(E)\right)^{2}+2(\operatorname{rk}(E))^{2} .
\end{aligned}
$$

Thus by Hodge index Theorem, we have

$$
\frac{H^{2}}{2}\left(\frac{H \operatorname{ch}_{1}(E)}{H^{2} \operatorname{rk}(E)}\right)^{2} \geq \frac{\operatorname{ch}_{1}(E)^{2}}{2 \operatorname{rk}(E)^{2}} \geq \frac{\operatorname{ch}_{2}(E)}{\operatorname{rk}(E)}+1-\frac{1}{\operatorname{rk}(E)^{2}}
$$


Fig. 2 Describing walls via $\operatorname{Ker} Z_{\beta, \alpha} \subset H^{*}(X, \mathbb{R})$

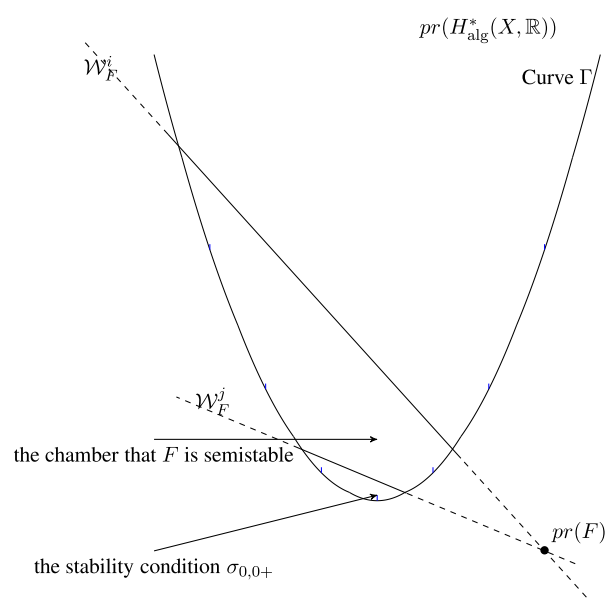

By the definition of $\gamma$ and Assumption (*), we have

$$
\gamma\left(\frac{H \operatorname{ch}_{1}(E)}{H^{2} \operatorname{rk}(E)}\right) \leq 1-\frac{1}{\operatorname{rk}(E)^{2}} .
$$

Together with (4), we have

$$
\Gamma\left(\frac{H \operatorname{ch}_{1}(E)}{H^{2} \operatorname{rk}(E)}\right) \geq \frac{H^{2}}{2}\left(\frac{H \operatorname{ch}_{1}(E)}{H^{2} \operatorname{rk}(E)}\right)^{2}-1+\frac{1}{\operatorname{rk}(E)^{2}} \geq \frac{\operatorname{ch}_{2}(E)}{\operatorname{rk}(E)}
$$

(b) The slope $v_{\beta, \alpha}(E)$ is just the slope of the line crossing points $(\beta, \alpha)$ and $\operatorname{pr}(E)$.

Proposition 2.4 [5, Proposition 9.3] Fix an object $F \in D^{b}(X)$. There exists a collection of line segments (walls) $\mathcal{W}_{F}^{i}$ in $\Gamma_{+}$with the following properties:

- the extension of each line segment passes through $\operatorname{pr}(F)$ if $\operatorname{rk}(F) \neq 0$; otherwise it has slope $\mathrm{ch}_{2}(F) H^{2} / H \mathrm{ch}_{1}(F)$;

- an endpoint of the segments is either on the curve $\Gamma$ or on the line segment through $\left(n, \frac{H^{2}}{2} n^{2}\right)$ to $\left(n, \frac{H^{2}}{2} n^{2}-1\right)$ for some $n \in \mathbb{Z}$ (see the remark below for more details);

- the $\sigma_{\beta, \alpha}-\left(\right.$ semi)stability or instability of $F$ does not change when $\sigma_{\beta, \alpha}$ changes between two consecutive walls.

- the object $F$ is strictly $\sigma_{\beta, \alpha}$-semistable if $(\beta, \alpha)$ is contained in one of the walls.

- if $F$ is $\sigma_{\beta, \alpha}$-semistable in one of the adjacent chambers to a wall, then it is unstable in the other adjacent chamber.

See Fig. 2 for a picture and [8] for more details.

Remark 2.5 In this paper, we will only apply Proposition 2.4 to an object $F=\iota_{*} E$ where $E$ is a slope semistable vector bundle on a curve $C \in|H|$. More precise descriptions for the walls of $\iota_{*} E$ are as follows.

- All walls of $\iota_{*} E$ are parallel segments with the same slope $\frac{\operatorname{ch}_{2}\left(\iota_{*} E\right)}{H \operatorname{ch}_{1}\left(\iota_{*} E\right)}$. 
- $\iota_{*} E$ is $\sigma_{0, a}$-semistable for $a \gg 0$.

- There is at most one wall $\mathcal{W}$ intersecting the line $\{(0, y) \mid y>0\}$. Indeed, if the wall contains passes through $\left(0, \alpha_{0}\right)$ for some $\alpha_{0}>0$, then the destabilizing subobject in $\operatorname{Coh}^{0}(S)$ will destabilize $\iota_{*} E$ for every $\alpha<\alpha_{0}$. So there is at most one $\alpha_{0}>0$ such that $\iota_{*} E$ is strictly semistable with respect to $\sigma_{0, \alpha_{0}}$.

- Suppose there is a wall $\mathcal{W}$ of $\iota_{*} E$ intersecting the line $\{(0, y) \mid y>0\}$. We will see in Lemma 3.1 below that the $x$-coordinates $\beta_{1}$ and $\beta_{2}$ of the endpoints of $\mathcal{W}$ satisfies $0<\beta_{2}-\beta_{1}<1$. In particular, both endpoints are on the curve $\Gamma$.

- There are also several walls irrelevant to our study. For each negative integer $n<0$ small enough, there is a 'tiny wall' with its 'right endpoint' at $\left(n, \frac{H^{2}}{2} n^{2}\right)$ and 'left endpoint' on curve $\Gamma$. These walls will never intersect the line $\{(0, y) \mid y>0\}$. So they are irrelevant to the HN factors of $\iota_{*} E$ at all. They are the only reason why we give several extra descriptions for the possible endpoints of walls.

\section{Bounds for the dimension of global sections}

In this section, we prove the first part of Theorem 1.1 which introduces a new upper bound for the dimension of global sections of vector bundles on a curve over a K3 surface. We always assume $X$ is a $\mathrm{K} 3$ surface with $\operatorname{Pic}(X)=\mathbb{Z} H$ and $C \in|H|$ is a smooth curve of genus $g$. We denote by $\iota: C \hookrightarrow X$ the embedding of the curve $C$ into $X$.

\subsection{The destabilizing wall for a stable vector bundle on the curve $C$}

Let $E$ be a slope semistable vector bundle on the curve $C$ of rank $r \geq 2$ and degree $d \in[0, r(g-1)]$. By [21, Theorem 3.11], the push-forward $\iota_{*} E$ is $\sigma_{\beta, \alpha}$-semistable for any $\beta \in \mathbb{R}$ and $\alpha$ sufficiently large. By Proposition 2.4, the walls for $\iota_{*} E$ are line segments of slope $\frac{d}{r}+1-g$. By Remark 2.5, there is at most one $\alpha>0$ such that $\iota_{*} E$ is 'destabilized' at $\sigma_{0, \alpha}$, in other words, $\iota_{*} E$ is strictly $\sigma_{0, \alpha}$-semistable and not $\sigma_{0, \alpha^{\prime}}$-semistable for every $0<\alpha^{\prime}<\alpha$. Suppose this is the case, in other words $\iota_{*} E$ becomes strictly semistable at the wall $\mathcal{W}$ which passes through $\sigma_{0, \alpha}$ for some $\alpha>0$. Denote the $x$-coordinates of the endpoints of the wall $\mathcal{W}$ as $\beta_{1}$ and $\beta_{2}$ for some $\beta_{1}<0<\beta_{2}$ (Fig. 3).

Lemma 3.1 Adopt notations as above, we have

$$
-1+\frac{d}{r H^{2}} \leq \beta_{1} \quad \text { and } \quad \beta_{2} \leq \frac{d}{r H^{2}} .
$$

Proof Let $0 \rightarrow F_{2} \rightarrow \iota_{*} E \rightarrow F_{1} \rightarrow 0$ in $\operatorname{Coh}^{0} X$ be the destabilizing sequence at the wall $\mathcal{W}$, then there is an exact sequence in $\operatorname{Coh} X$ : 


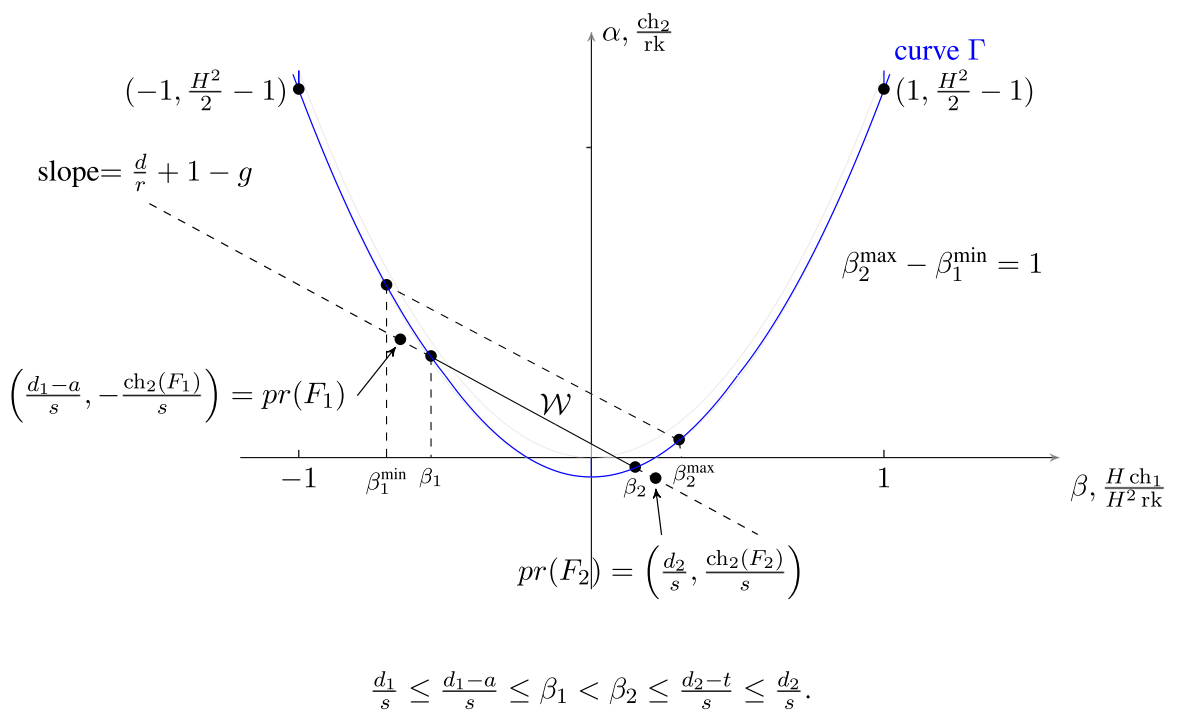

Fig. 3 Cartoon for the destabilizing wall $\mathcal{W}$ of $\iota_{*} E$

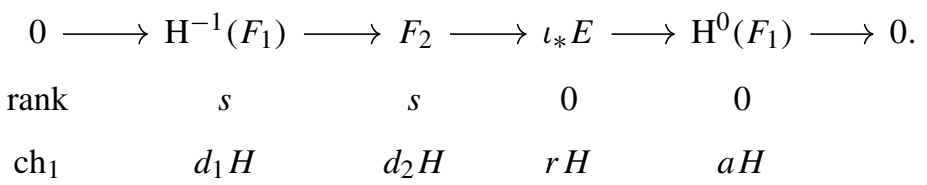

If $s=0$, then since $F_{2}$ and $\iota_{*} E$ have the same phase with respect to $\sigma_{0, \alpha}$, it follows that $\operatorname{ch}\left(\iota_{*} E\right)=\left(\frac{r}{d_{2}}\right) \operatorname{ch}\left(F_{2}\right)$, so that $F_{2}$ cannot make a wall for $\iota_{*} E$. Thus, we may assume $s>0$. Let $T\left(F_{2}\right)$ be the maximal torsion subsheaf of $F_{2}$ and $\operatorname{ch}_{1}\left(T\left(F_{2}\right)\right)=$ $t H$. Since $E$ is of rank $r$, to make the sequence exact at the term $\iota_{*} E$, we must have

$$
r-a \leq \operatorname{rank}\left(\iota^{*} T\left(F_{2}\right)\right)+\operatorname{rank}\left(\iota^{*} F_{2} / T\left(F_{2}\right)\right)=s+t .
$$

Therefore,

$$
\frac{H \operatorname{ch}_{1}\left(F_{2} / T\left(F_{2}\right)\right)}{s H^{2}}-\frac{H \operatorname{ch}_{1}\left(H^{-1}\left(F_{1}\right)\right)}{s H^{2}}=\frac{d_{2}-t-d_{1}}{s}=\frac{r-a-t}{s} \leq 1 .
$$

By Proposition 2.4, the object $F_{1}$ is semistable of the same phase as $\iota_{*} E$ along the line segment $\mathcal{W}$, in particular if $-1<\beta_{1}$, it is in the heart $\mathrm{Coh}^{\beta_{1}+\epsilon} X$ where $\epsilon \rightarrow 0^{+}$. Thus by definition of the tilting heart,

$$
\frac{H \operatorname{ch}_{1}\left(H^{-1}\left(F_{1}\right)\right)}{H^{2} s}=\frac{d_{1}}{s} \leq \beta_{1} .
$$


By similar reasoning for $F_{2} / T\left(F_{2}\right)$, it follows from the definition of the tilting heart that

$$
\frac{H \operatorname{ch}_{1}\left(F_{2} / T\left(F_{2}\right)\right)}{H^{2} s} \geq \beta_{2} .
$$

Therefore inequality (5) and definition of imply that

$$
0<\beta_{2} \leq \frac{H \operatorname{ch}_{1}\left(F_{2} / T\left(F_{2}\right)\right)}{H^{2} s} \leq 1+\beta_{1}<1 .
$$

In particular, $\beta_{1}>-1, \beta_{2}<1$, and $\beta_{2}-\beta_{1} \leq 1$.

By the second property of Proposition 2.4, the slope of $\mathcal{W}$ as a line in the projection $\operatorname{pr}\left(H_{\text {alg }}^{*}(X, \mathbb{R})\right)$ is

$$
\frac{\Gamma\left(\beta_{2}\right)-\Gamma\left(\beta_{1}\right)}{\beta_{2}-\beta_{1}}=\frac{H^{2} \operatorname{ch}_{2}\left(\iota_{*} E\right)}{H \operatorname{ch}_{1}\left(\iota_{*} E\right)}=-\frac{H^{2}}{2}+\frac{d}{r} .
$$

It is not hard to see that $\beta_{2}$ (respectively $\beta_{1}$ ) reaches its maximum $\beta_{2}^{\max }$ (respectively minimum $\beta_{1}^{\mathrm{min}}$ ) when $\beta_{2}-\beta_{1}=1$. Substitute this to (9), we get

$$
\Gamma\left(\beta_{2}^{\max }\right)-\Gamma\left(\beta_{2}^{\max }-1\right)=-\frac{H^{2}}{2}+\frac{d}{r} .
$$

Since $0 \leq d \leq r(g-1)$, slope of $\mathcal{W}$ is not positive, thus $0<\beta_{2}^{\max } \leq \frac{1}{2}$ and by Definition (2.1),

$$
\begin{gathered}
\Gamma\left(\beta_{2}^{\max }\right)=\frac{H^{2}}{2}\left(\beta_{2}^{\max }\right)^{2}-\left(1-\left(\beta_{2}^{\max }\right)^{2}\right), \\
\Gamma\left(\beta_{2}^{\max }-1\right)=\frac{H^{2}}{2}\left(\beta_{2}^{\max }-1\right)^{2}-\left(1-\left(\beta_{2}^{\max }\right)^{2}\right)
\end{gathered}
$$

Substituting back into the Eq. (10) gives $\beta_{2}^{\max }=\frac{d}{r H^{2}}$ and $\beta_{1}^{\min }=\frac{d}{r H^{2}}-1$.

We need the following description for the first wall in details.

\section{Lemma 3.2 Adopt notations from Lemma 3.1.}

(a) If $r \leq s=\operatorname{rk}\left(F_{2}\right)$ and $\operatorname{ch}_{1}\left(F_{2} / T\left(F_{2}\right)\right)=H$, we have $-1+\frac{1}{r} \leq \beta_{1}$. Otherwise $-1+\frac{1}{r-1} \leq \beta_{1}$.

(b) When $0 \leq d \leq \min \{2 g-2+r, r(g-1)\}$ and $g \geq r^{2}$, we have $\beta_{2} \leq \frac{1}{r}$.

(c) When $r=3$, we either have the Chern characters $\operatorname{ch}\left(F_{2}\right)=(3, H,-)$ or $\beta_{1} \geq$ $-\frac{1}{2}$.

Proof Adopt the notations as in the proof of Lemma 3.1.

(a) By inequality (7), we know

$$
\frac{H \operatorname{ch}_{1}\left(F_{2} / T\left(F_{2}\right)\right)}{H^{2}} \geq s \beta_{2}>0 .
$$


Recall that $\frac{H \mathrm{ch}_{1}\left(F_{2} / T\left(F_{2}\right)\right)}{H^{2}} \in \mathbb{N}$ by Assumption $(*)$.

- If $s=\operatorname{rk}\left(F_{2}\right)<r$, then $\frac{H \operatorname{ch}_{1}\left(F_{2} / T\left(F_{2}\right)\right)}{H^{2} s} \geq \frac{1}{r-1}$. Thus (8) gives

$$
\frac{1}{r-1} \leq \frac{H \operatorname{ch}_{1}\left(F_{2} / T\left(F_{2}\right)\right)}{H^{2} s} \leq 1+\beta_{1}
$$

comparing the first and the last sentences implies the claim.

- If $r \leq s$, inequality (6) gives

$$
\frac{H \operatorname{ch}_{1}\left(H^{-1}\left(F_{1}\right)\right)}{H^{2} r} \leq \frac{H \operatorname{ch}_{1}\left(H^{-1}\left(F_{1}\right)\right)}{H^{2} s} \leq \beta_{1}<0 .
$$

Taking $\mathrm{ch}_{1}$ from the destabilizing sequence gives

$$
\begin{gathered}
\frac{H \operatorname{ch}_{1}\left(H^{-1}\left(F_{1}\right)\right)}{H^{2}}=-r+\frac{H \operatorname{ch}_{1}\left(F_{2} / T\left(F_{2}\right)\right)}{H^{2}} \\
+\frac{H \operatorname{ch}_{1}\left(T\left(F_{2}\right)\right)}{H^{2}}+\frac{H \operatorname{ch}_{1}\left(H^{0}\left(F_{1}\right)\right)}{H^{2}}
\end{gathered}
$$

Since $H^{0}\left(F_{2}\right)$ is a torsion sheaf $H \operatorname{ch}_{1}\left(H^{0}\left(F_{1}\right)\right) \geq 0$. Also by $(11), \frac{H \operatorname{ch}_{1}\left(F_{2} / T\left(F_{2}\right)\right)}{H^{2}} \geq$ 1. If $\frac{H \operatorname{ch}_{1}\left(F_{2} / T\left(F_{2}\right)\right)}{H^{2}} \geq 2$, then $\frac{H \operatorname{ch}_{1}\left(H^{-1}\left(F_{1}\right)\right)}{H^{2}} \geq-r+2$ and by (12)

$$
-1+\frac{1}{r-1} \leq \frac{-r+2}{r} \leq \frac{H \operatorname{ch}_{1}\left(H^{-1}\left(F_{1}\right)\right)}{H^{2} r} \leq \beta_{1}
$$

as claimed. If $\frac{H \operatorname{ch}_{1}\left(F_{2} / T\left(F_{2}\right)\right)}{H^{2}}=1$, then $\frac{H \operatorname{ch}_{1}\left(H^{-1}\left(F_{1}\right)\right)}{H^{2}} \geq-r+1$ and by (12)

$$
\frac{-r+1}{r} \leq \frac{H \operatorname{ch}_{1}\left(H^{-1}\left(F_{1}\right)\right)}{H^{2} r} \leq \beta_{1}
$$

This finishes the proof of part (a).

(b) If $r \leq s=\operatorname{rk}\left(F_{2}\right)$ and $\operatorname{ch}_{1}\left(F_{2} / T\left(F_{2}\right)\right)=H$, then by (11), $\beta_{2} \leq \frac{H \operatorname{ch}_{1}\left(F_{2} / T\left(F_{2}\right)\right)}{H^{2} s} \leq$ $\frac{1}{r}$ and the claim follows. Thus we may assume otherwise, so part $(a)$ gives $\beta_{1} \geq \frac{2-r}{r-1}$. When $r \leq 2, d \leq 2(g-1)=H^{2}$. By Lemma 3.1, $\beta_{2} \leq \frac{d}{r H^{2}} \leq \frac{1}{r}$. So the statement holds.

We may assume $r \geq 3$. Suppose for a contradiction that $\beta_{2}>\frac{1}{r}$, then by definition $\Gamma$, the slope of the line connecting $\left(\frac{1}{r}, \Gamma\left(\frac{1}{r}\right)\right)$ and $\left(\frac{2-r}{r-1}, \Gamma\left(\frac{2-r}{r-1}\right)\right)$ is less than slope of the line connecting $\left(\beta_{2}, \Gamma\left(\beta_{2}\right)\right)$ and $\left(\beta_{1} \Gamma\left(\beta_{1}\right)\right)$, in other words,

$$
\frac{\Gamma\left(\frac{1}{r}\right)-\Gamma\left(\frac{2-r}{r-1}\right)}{\frac{1}{r}-\frac{2-r}{r-1}}<\frac{\Gamma\left(\beta_{2}\right)-\Gamma\left(\beta_{1}\right)}{\beta_{2}-\beta_{1}}=\text { the slope of } \mathcal{W}=-\frac{H^{2}}{2}+\frac{d}{r}
$$


Since $0<\frac{1}{r-1} \leq \frac{1}{2}$, we have $-1<-1+\frac{1}{r-1} \leq-\frac{1}{2}$, the definition (2.1) of $\Gamma$ gives

$$
\Gamma\left(\frac{1}{r}\right)=\frac{H^{2}}{2} \cdot \frac{1}{r^{2}}-1+\frac{1}{r^{2}}, \quad \Gamma\left(\frac{2-r}{r-1}\right)=\frac{H^{2}}{2}\left(\frac{2-r}{r-1}\right)^{2}-1+\frac{1}{(r-1)^{2}}
$$

Substitute them into the left hand side of (14):

$$
\begin{aligned}
\frac{\Gamma\left(\frac{1}{r}\right)-\Gamma\left(\frac{2-r}{r-1}\right)}{\frac{1}{r}-\frac{2-r}{r-1}} & =\frac{H^{2}}{2}\left(\frac{1}{r}+\frac{2-r}{r-1}\right)+\frac{\frac{1}{r^{2}}-\frac{1}{(r-1)^{2}}}{\frac{1}{r}-\frac{2-r}{r-1}} \\
& =\frac{H^{2}}{2}\left(-1+\frac{2}{r}+\frac{1}{r(r-1)}\right)-\frac{2 r-1}{r(r-1)\left(r^{2}-r-1\right)} \\
& =-\frac{H^{2}}{2}+\frac{H^{2}}{r}+\frac{1}{r(r-1)}\left(\frac{H^{2}}{2}-\frac{2 r-1}{r^{2}-r-1}\right) .
\end{aligned}
$$

Since $g \geq r^{2}$, we get $g>r(r-1)+1+\frac{2 r-1}{r^{2}-r-1}$ because $r>1+\frac{2 r-1}{r^{2}-r-1}$ for $r \geq 3$. This implies

$$
\frac{H^{2}}{2}-\frac{2 r-1}{r^{2}-r-1}>r(r-1)
$$

Therefore (15) gives

$$
\frac{\Gamma\left(\frac{1}{r}\right)-\Gamma\left(\frac{2-r}{r-1}\right)}{\frac{1}{r}-\frac{2-r}{r-1}}>-\frac{H^{2}}{2}+\frac{H^{2}}{r}+1 \geq-\frac{H^{2}}{2}+\frac{d}{r}
$$

with the last inequality is given by the assumption $d \leq H^{2}+r$. So this contradicts (14).

(c) By part (a), we may assume $r=3 \leq s=\operatorname{rk}\left(F_{2}\right)$ and $\operatorname{ch}_{1}\left(F_{2} / T\left(F_{2}\right)\right)=H$. Substituting $r=3$ and $\operatorname{ch}_{1}\left(F_{2} / T\left(F_{2}\right)\right)=H$ in (13) implies that $-2 \leq \frac{H \operatorname{ch}_{1}\left(H^{-1}\left(F_{1}\right)\right)}{H^{2}}$. On the other hand, (12) gives $H \operatorname{ch}_{1}\left(H^{-1}\left(F_{1}\right)\right)<0$. If $\operatorname{ch}_{1}\left(H^{-1}\left(F_{1}\right)\right)=-H$, or $\operatorname{ch}_{1}\left(H^{-1}\left(F_{1}\right)\right)=-2 H$ and $s \geq 4$, then (12) gives $-\frac{1}{2} \leq \beta_{1}$ as claimed. Hence we can assume $\operatorname{ch}_{1}\left(H^{-1}\left(F_{1}\right)\right)=-2 H$ and $s=3$. Thus (13) gives $\operatorname{ch}_{1}\left(T\left(F_{2}\right)\right)=0$, so $\operatorname{ch}_{1}\left(F_{2}\right)=H$ as required.

\subsection{An upper bound on the dimension of global sections}

We first recall the result in [8, Section 3]. Define the function $\bar{Z}: K(X) \rightarrow \mathbb{C}$ as

$$
\bar{Z}(F)=\operatorname{ch}_{2}(F)+i \frac{\operatorname{ch}_{1}(F) \cdot H}{H^{2}} .
$$


We also define the following non-standard norm on $\mathbb{C}$ :

$$
\|x+i y\|=\sqrt{x^{2}+\left(2 H^{2}+4\right) y^{2}} .
$$

The next proposition bounds the dimension of global sections of objects in terms of the length of a polygon.

Proposition 3.3 [8, Proposition 3.4] Let $F \in \operatorname{Coh}^{0} X$ be an object which has no subobject $F^{\prime} \subset F$ with $\operatorname{ch}_{1}\left(F^{\prime}\right)=0$.

(a) There exists $\epsilon>0$ such that the Harder-Narasimhan filtration of $F$ is a fixed sequence

$$
0=\tilde{E}_{0} \subset \tilde{E}_{1} \subset \cdots \subset \tilde{E}_{n-1} \subset \tilde{E}_{n}=F
$$

for all stability conditions $\sigma_{0, \alpha}$ where $0<\alpha<\epsilon$.

(b) Let $p_{i}:=\bar{Z}\left(\tilde{E}_{i}\right)$ for $0 \leq i \leq n$, then

$$
h^{0}(X, F) \leq \frac{\chi(F)}{2}+\frac{1}{2} \sum_{i=1}^{n}\left\lfloor\left\|p_{i} p_{i-1}\right\|\right\rfloor
$$

where $\left\lfloor\left\|p_{i} p_{i-1}\right\|\right\rfloor$ is the integer part of the length of the line segment $p_{i} p_{i-1}$ and $\chi(F)$ is the Euler characteristic of $F$.

Proof In the notations of [8], $\frac{H^{2}}{2} w^{2}-1$ is equal to our $\alpha$, so part (a) follows from [8, Proposition 3.3 (a)]. By [8, Lemma 3.2],

$$
\begin{aligned}
h^{0}\left(\tilde{E}_{i} / \tilde{E}_{i-1}\right) & \leq\left\lfloor\frac{\chi\left(\tilde{E}_{i} / \tilde{E}_{i-1}\right)}{2}+\frac{\left\|p_{i} p_{i-1}\right\|}{2}\right\rfloor \\
& =\left\lfloor\frac{\chi\left(\tilde{E}_{i} / \tilde{E}_{i-1}\right)}{2}+\frac{\left\lfloor\left\|p_{i} p_{i-1}\right\|\right\rfloor}{2}+\frac{\left\|p_{i} p_{i-1}\right\|-\left\lfloor\left\|p_{i} p_{i-1}\right\|\right\rfloor}{2}\right\rfloor \\
& \leq \frac{\chi\left(\tilde{E}_{i} / \tilde{E}_{i-1}\right)}{2}+\frac{\left\lfloor\left\|p_{i} p_{i-1}\right\|\right\rfloor}{2}
\end{aligned}
$$

where the last inequality following from the following two cases:

(a) If both $\chi\left(\tilde{E}_{i} / \tilde{E}_{i-1}\right)$ and $\left\lfloor\left\|p_{i} p_{i-1}\right\|\right\rfloor$ are even or odd, the claim is trivial because $\left\|p_{i} p_{i-1}\right\|-\left\lfloor\left\|p_{i} p_{i-1}\right\|\right\rfloor<1$.

(b) If either $\chi\left(\tilde{E}_{i} / \tilde{E}_{i-1}\right)$ or $\left\lfloor\left\|p_{i} p_{i-1}\right\|\right\rfloor$ is odd, then since $\frac{1}{2}+\frac{\left\|p_{i} p_{i-1}\right\|-\left\lfloor\left\|p_{i} p_{i-1}\right\|\right\rfloor}{2}<1$ the claim follows.

Finally by summing up over all stable factors one gets

$$
h^{0}(F) \leq \sum_{i=1}^{n} h^{0}\left(\tilde{E}_{i} / \tilde{E}_{i-1}\right) \leq \frac{1}{2} \sum_{i=1}^{n} \chi\left(\tilde{E}_{i} / \tilde{E}_{i-1}\right)+\frac{1}{2} \sum_{i=1}^{n}\left\lfloor\left\|p_{i} p_{i-1}\right\|\right\rfloor
$$


Fig. 4 The polygon $P_{l_{*} E}$ is inside the triangle $o p q$

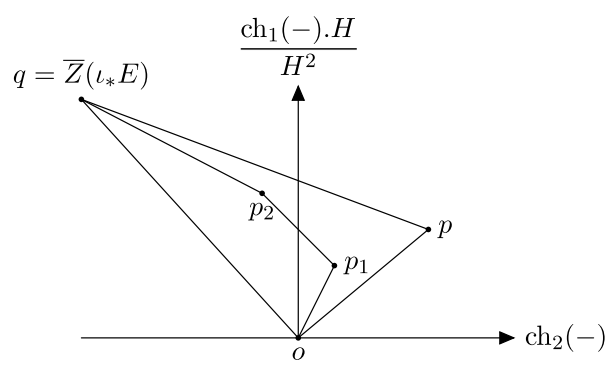

$$
=\frac{\chi(F)}{2}+\frac{1}{2} \sum_{i=1}^{n}\left\lfloor\left\|p_{i} p_{i-1}\right\|\right\rfloor .
$$

We denote by $P_{F}$ the polygon with the extremal points $\left\{p_{0}, p_{1}, \ldots, p_{n}\right\}$ which is a convex polygon.

Let $E$ be a slope semistable rank $r$-vector bundle on the curve $C$ of degree $d$.

Proposition 3.3 implies that there exists $\epsilon>0$ such that the Harder-Narasimhan filtration of $\iota_{*} E$ with respect to the stability condition $\sigma_{0, \alpha}$ for positive $\alpha<\epsilon$ is a fixed sequence

$$
0=\tilde{E}_{0} \subset \tilde{E}_{1} \subset \cdots \subset \tilde{E}_{n-1} \subset \tilde{E}_{n}=\iota_{*} E
$$

Consider the triangle $o p q$ where $o$ is the origin, $q=\bar{Z}\left(\iota_{*} E\right)$, the slope of $\overline{o p}$ is equal to $\beta_{2} / \Gamma\left(\beta_{2}\right)$ and the slope of $\overline{p q}$ is $\beta_{1} / \Gamma\left(\beta_{1}\right)$, where the real numbers $\beta_{1}$ and $\beta_{2}$ are defined as in Lemma 3.1 (Fig. 4).

Lemma 3.4 The polygon $P_{\iota_{*} E}$ is contained in the triangle opq.

Proof If $\iota_{*} E$ is $\sigma_{0, \alpha}$-semistable where $\alpha \rightarrow 0^{+}$, then the polygon $P_{\iota_{*} E}$ is just the line segment $o q$ and the claim follows. Thus, we may assume $\iota_{*} E$ is not $\sigma_{0, \alpha}$-semistable where $\alpha \rightarrow 0^{+}$. Since the polygon $P_{l_{*} E}$ is convex, it suffices to show that

$$
\frac{H^{2} \operatorname{ch}_{2}\left(\tilde{E}_{1}\right)}{H \operatorname{ch}_{1}\left(\tilde{E}_{1}\right)} \leq \frac{\Gamma\left(\beta_{2}\right)}{\beta_{2}} \quad \text { and } \quad \frac{\Gamma\left(\beta_{1}\right)}{\beta_{1}} \leq \frac{H^{2} \operatorname{ch}_{2}\left(\iota_{*} E / \tilde{E}_{n-1}\right)}{H \operatorname{ch}_{1}\left(\iota_{*} E / \tilde{E}_{n-1}\right)}
$$

The phase of the subobject $\tilde{E}_{1}$ in the Harder-Narasimhan filtration is bigger than phase of $\iota_{*} E$ at the stability condition $\sigma_{0, \alpha}$ where $\alpha \rightarrow 0^{+}$. Therefore there are stability condition between large volume limit $\left(\sigma_{\beta, \alpha}\right.$ where $\left.\alpha \rightarrow \infty\right)$ and the stability conditions $\sigma_{0, \alpha}$ where $\alpha \rightarrow 0^{+}$such that $\tilde{E}_{1}$ and $\iota_{*} E$ have the same phase. Proposition 2.4 implies that these stability conditions are on a line segment $L$ whose extension passes through the point $\operatorname{pr}\left(\tilde{E}_{1}\right)$. Note that $\operatorname{rk}\left(\tilde{E}_{1}\right) \neq 0$ by the same argument as that in the beginning of the proof of Lemma 3.1. The line $L$ is lower than the wall $\mathcal{W}$ for $\iota_{*} E$ since otherwise $\iota_{*} E$ will already become strictly semistable on $L$, see Fig. 5 . 


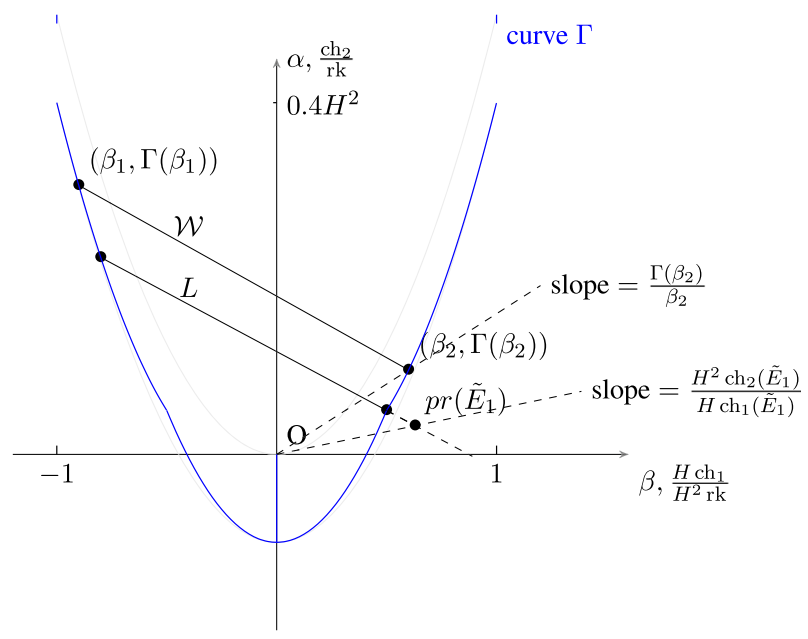

Fig. 5 Comparing slopes

Since $\tilde{E}_{1}$ is $\sigma_{0, \alpha}$-semistable for some $\alpha>0$, the point $\operatorname{pr}\left(\tilde{E}_{1}\right)$ is not in $\Gamma_{+}$by Remark 2.3. Therefore, $\operatorname{pr}\left(\tilde{E}_{1}\right)$ is on the dashed part of the line $L$ and the first claim follows. By a similar argument one can show the second claim for $\iota_{*} E / \tilde{E}_{n-1}$.

We are now ready to prove the bound for the dimension of global sections of the semistable vector bundle $E$.

Proof for the first part of Theorem 1.1 Consider the triangle $o p^{\prime} q$ where the slope of $o p^{\prime}$ is

$$
\frac{\frac{d}{r H^{2}}}{\Gamma\left(\frac{d}{r H^{2}}\right)}=\frac{\frac{d}{r H^{2}}}{\frac{d^{2}}{2 r^{2} H^{2}}-1+\frac{d^{2}}{r^{2}\left(H^{2}\right)^{2}}}
$$

and the slope of $p^{\prime} q$ is

$$
\frac{\frac{d}{r H^{2}}-1}{\Gamma\left(\frac{d}{r H^{2}}-1\right)}=\frac{\frac{d}{r H^{2}}-1}{\frac{H^{2}}{2}\left(\frac{d}{r H^{2}}-1\right)^{2}-1+\frac{d^{2}}{r^{2}\left(H^{2}\right)^{2}}} .
$$

Lemma 3.1 implies that the triangle $o p q$ is inside the triangle $o p^{\prime} q$, so by Lemma 3.4 the polygon $P_{l_{*} E}$ is also inside the triangle $o p^{\prime} q$. By a direct computation, one can show that the point

$$
p^{\prime}=\left(\frac{d^{2} g}{\left(H^{2}\right)^{2} r}-r, \frac{d}{H^{2}}\right) .
$$

Now Proposition 3.3, part $(b)$ gives 


$$
\begin{aligned}
h^{0}\left(X, \iota_{*} E\right) \leq & \frac{\chi\left(\iota_{*} E\right)}{2}+\frac{1}{2} \sum_{i=1}^{n}\left\|p_{i} p_{i-1}\right\| \\
\leq & \frac{\chi\left(\iota_{*} E\right)}{2}+\frac{1}{2}\left(\left\|o p^{\prime}\right\|+\left\|p^{\prime} q\right\|\right) \\
= & \frac{1}{2}(r(1-g)+d)+\frac{1}{2} \sqrt{\left(\frac{d^{2} g}{\left(H^{2}\right)^{2} r}-r\right)^{2}+4 g\left(\frac{d}{H^{2}}\right)^{2}} \\
& +\frac{1}{2} \sqrt{\left(r(g-1)-d+\frac{d^{2} g}{\left(H^{2}\right)^{2} r}-r\right)^{2}+4 g\left(r-\frac{d}{H^{2}}\right)^{2}} \\
= & \frac{1}{2}(r(1-g)+d)+\frac{1}{2}\left(\frac{d^{2} g}{\left(H^{2}\right)^{2} r}+r\right) \\
& +\frac{1}{2}\left(r(g-1)-d+\frac{d^{2} g}{\left(H^{2}\right)^{2} r}+r+\delta\right),
\end{aligned}
$$

where the last equality holds for the non-negative solution $\delta$ to the following equation.

$$
\begin{aligned}
(r+\delta)^{2}+2(r+\delta) & \left(r(g-1)-d+\frac{d^{2} g}{\left(H^{2}\right)^{2} r}\right) \\
= & r^{2}-2 r\left(r(g-1)-d+\frac{d^{2} g}{\left(H^{2}\right)^{2} r}\right) \\
& +4 g\left(r-\frac{d}{H^{2}}\right)^{2} .
\end{aligned}
$$

This is equivalent to

$$
\delta^{2}+2 r \delta+2 \delta\left(r(g-1)-d+\frac{d^{2} g}{\left(H^{2}\right)^{2} r}\right)=4 r^{2}-\frac{4 d r}{g-1} .
$$

Now we will show that $\delta<\frac{2 r}{g}$. Since $0 \leq r(g-1)-d$, the function $f(x):=x^{2}+$ $2 r x+2 x\left(r(g-1)-d+\frac{d^{2} g}{\left(H^{2}\right)^{2} r}\right)$ is increasing for $x>0$. Moreover,

$$
\begin{aligned}
f\left(\frac{2 r}{g}\right) & =\frac{4 r^{2}}{g^{2}}+\frac{4 r^{2}}{g}+\frac{4 r}{g}\left(r(g-1)-d+\frac{d^{2} g}{\left(H^{2}\right)^{2} r}\right) \\
& =4 r^{2}+\frac{4 r^{2}}{g^{2}}-\frac{4 r d}{g}+\frac{d^{2}}{(g-1)^{2}} \\
& =4 r^{2}-\frac{4 d r}{g-1}+\left(\frac{2 r}{g}+\frac{d}{g-1}\right)^{2} \\
& >4 r^{2}-\frac{4 d r}{g-1} .
\end{aligned}
$$


Hence (17) shows $f(\delta)<f\left(\frac{2 r}{g}\right)$ which gives $\delta<\frac{2 r}{g}$. Applying this back into (16) implies

$$
\begin{aligned}
h^{0}(C, E)< & \frac{1}{2}(r(1-g)+d)+\frac{1}{2}\left(\frac{d^{2} g}{\left(H^{2}\right)^{2} r}+r\right) \\
& +\frac{1}{2}\left(r(g-1)-d+\frac{d^{2} g}{\left(H^{2}\right)^{2} r}+r+\frac{2 r}{g}\right) \\
= & r+\frac{g}{4 r(g-1)^{2}} d^{2}+\frac{r}{g} .
\end{aligned}
$$

Remark 3.5 The bound for $h^{0}(C, E)$ in Theorem 1.1 is not far from the sharp bound. Let $k$ be an integer in $[1, r]$, denote $t=\operatorname{gcd}(r, k)$. When $d=2 k(g-1)$ such that $g \geq\left(\frac{r}{t}\right)^{2}+2$, there exists a stable vector bundle $F$ on $X$ with Chern characters:

$$
\left(\operatorname{rk}(F), \operatorname{ch}_{1}(F), \operatorname{ch}_{2}(F)\right)=\left(\frac{r}{t}, \frac{k}{t} H,\left\lfloor\frac{t}{r}+\frac{k^{2}}{r t}(g-1)\right\rfloor-\frac{r}{t}\right) .
$$

When $k=r, F$ is a line bundle, so the restriction $\left.F^{\oplus t}\right|_{C}$ is semistable.

When $k<r$, the rank of $F$ is greater than 1 . Since $\operatorname{Pic}(X)=\mathbb{Z} . H,[9$, Proposition 4.6] implies $\left.F\right|_{C}$ is semistable if

$$
\operatorname{rk}(F)(\operatorname{rk}(F)-1) \widetilde{\Delta}(F)+\frac{1}{\operatorname{rk}(F)(\operatorname{rk}(F)-1)} \leq 1,
$$

where

$$
\widetilde{\Delta}(F)=\frac{\left(\operatorname{ch}_{1}(F) H\right)^{2}-2 H^{2} \operatorname{rk}(F) \operatorname{ch}_{2}(F)}{\left(H^{2} \operatorname{rk}(F)\right)^{2}}=\frac{k^{2}}{r^{2}}-\frac{t}{r(g-1)} \operatorname{ch}_{2}(F) .
$$

We have

$$
\begin{aligned}
\widetilde{\Delta}(F) & <\frac{k^{2}}{r^{2}}-\frac{t}{r(g-1)}\left(\frac{t}{r}+\frac{k^{2}}{r t}(g-1)-1-\frac{r}{t}\right) \\
& \leq \frac{\frac{t}{r}}{\left(\frac{r}{t}\right)^{2}+1}\left(1+\frac{r}{t}-\frac{t}{r}\right) \\
& =\frac{1}{\operatorname{rk}(F)\left(\operatorname{rk}(F)^{2}+1\right)}\left(1+\operatorname{rk}(F)-\frac{1}{\operatorname{rk}(F)}\right) .
\end{aligned}
$$

Thus (18) clearly holds and the restriction $\left.F^{\oplus t}\right|_{C}$ is semistable with rank $r$, degree $2 k(g-1)$ and dimension of global sections

$$
h^{0}\left(C,\left.F^{\oplus t}\right|_{C}\right)=h^{0}\left(X, F^{\oplus t}\right)=t\left\lfloor\frac{t}{r}+\frac{k^{2}}{r t}(g-1)\right\rfloor+r .
$$


If the $\lfloor\cdot\rfloor$ function can be dropped for free, the formula can be simplified as

$$
r+\frac{g}{4 r(g-1)^{2}} d^{2}+\frac{t^{2}-k^{2}}{r} .
$$

Corollary 3.6 Let $(X, H)$ be a smooth polarized K3 surface satisfying Assumption (*), and let $C$ be a smooth curve of genus $g$ in the linear system $|H|$. Let $E$ be a slope semistable rank $r$-vector bundle of degree $d$ on the curve $C$ such that $d \leq r(g-1)$. Then Cliff $(E)>\frac{d}{r}-\frac{d^{2} g}{2 r^{2}(g-1)^{2}}-\frac{2}{g}$. When $g \geq 7$, we have

$$
\operatorname{Cliff}_{r}(C)>2 \sqrt{g-1}-2-\frac{2 \sqrt{g-1}}{g} .
$$

Proof The bound for Cliff $(E)$ is by substituting the bounds of $h^{0}(C, E)$ into the formula of Clifford index. By the first part of Theorem 1.1, if $h^{0}(C, E) \geq 2 r$, then

$$
r+\frac{g}{4 r(g-1)^{2}} d^{2}+\frac{r}{g}>h^{0}(C, E) \geq 2 r
$$

This implies $\frac{g}{4 r(g-1)^{2}} d^{2}>r \frac{g-1}{g}$ which is equivalent to $d>\frac{2 r(g-1)^{\frac{3}{2}}}{g}$. Consider the function $f(d)=\frac{d}{r}-\frac{d^{2} g}{2 r^{2}(g-1)^{2}}-\frac{2}{g}$, it reaches the maximum when $d=\frac{r(g-1)^{2}}{g}$. When $g \geq 7$, the value $\frac{r(g-1)^{2}}{g}$ is in the range of $d \in\left[\frac{2 r(g-1)^{\frac{3}{2}}}{g}, r(g-1)\right]$. To know at which boundary $f(d)$ reaches its minimum, we compare the distances from the two boundaries to $\frac{r(g-1)^{2}}{g}$.

$$
\begin{gathered}
\frac{r(g-1)^{2}}{g}-\frac{2 r(g-1)^{\frac{3}{2}}}{g} \geq r(g-1)-\frac{r(g-1)^{2}}{g} \\
\Longleftrightarrow g-1-2 \sqrt{g-1} \geq 1 \Longleftrightarrow g \geq 7 .
\end{gathered}
$$

Therefore, the function $f(d)$ reaches its minimum at the left boundary. In particular,

$$
\begin{aligned}
& \operatorname{Cliff}_{r}(C)>f\left(\frac{2 r(g-1)^{\frac{3}{2}}}{g}\right)=\frac{2 r(g-1)^{\frac{3}{2}}}{g r}-\frac{4 r^{2}(g-1)^{3}}{2 r^{2} g(g-1)^{2}}-\frac{2}{g} \\
& =2 \sqrt{g-1}-2-\frac{2 \sqrt{g-1}}{g}
\end{aligned}
$$

for any $r$. 


\section{Higher rank Clifford indices}

In this section, we compute higher rank Clifford indices of curves over K3 surfaces and prove the second part of Theorem 1.1.

\subsection{Picard number one case}

We assume $X$ is a K3 surface with $\operatorname{Pic}(X)=\mathbb{Z} H$ and $C \in|H|$ is a smooth curve of genus $g$. Denote by $\iota: C \hookrightarrow X$ the embedding of the curve $C$ into $X$. We first briefly recall the result in [9], which constructs semistable vector bundles on $C$ by restricting vector bundles on $X$ with low discriminant. By [2, Theorem 2.15], there exists a slope stable sheaf $\tilde{E}_{r}$ on $X$ with Chern character $\left(r, H,\left\lfloor\frac{g}{r}\right\rfloor-r\right)$. Define $E_{r}:=\left.\tilde{E}_{r}\right|_{C}$.

Theorem 4.1 [9, Theorem 1.2] Assume $g \geq \max \left\{r^{2}, 6\right\}$ and $r \geq 2$, then the sheaf $E_{r}$ is a semistable vector bundle on $C$ with $h^{0}\left(C, E_{r}\right) \geq 2 r$ and

$$
\operatorname{Cliff}\left(E_{r}\right) \leq \frac{2}{r}(g-1)-\frac{2}{r}\left\lfloor\frac{g}{r}\right\rfloor
$$

Proof The stable sheaf $\tilde{E}_{r}$ is locally-free, otherwise, the double dual $F=\tilde{E}_{r}^{\vee \vee}$ is slope stable with Chern characters $\left(r, H,\left\lfloor\frac{g}{r}\right\rfloor-s\right)$ for some integer $s \leq r-1$. Yet $-\chi(F, F)=H^{2}-2 r\left(\left\lfloor\frac{g}{r}\right\rfloor-s\right)-2 r^{2}<-2$. This contradicts [2, Theorem 2.15]. Thus by the assumption on $r$ and $g$, [9, Theorem 1.2] implies that $E_{r}$ is slope semistable on $C$ and $h^{0}(C, E) \geq 2 r$. As $\operatorname{deg}\left(E_{r}\right)=c_{1}\left(\tilde{E}_{r}\right) H=2(g-1)$, by a direct computation, Cliff $\left(E_{r}\right) \leq \frac{2}{r}(g-1)-\frac{2}{r}\left\lfloor\frac{g}{r}\right\rfloor$.

We now prove the Clifford index of $E_{r}$ is indeed the minimum of Clifford index of any semistable vector bundle $E$ with rank $r$, degree $d$ and $h^{0}(E) \geq 2 r$. This will involve several different cases.

Proof of the second part of Theorem 1.1 for $r \geq 4$. Let $E$ be a semistable rank $r$-vector bundle of degree $d \leq r(g-1)$ on the curve $C$. By Theorem 4.1, it suffices to show that either $h^{0}(E)<\overline{2} r$ or $\operatorname{Cliff}(E) \geq \frac{2}{r}(g-1)-\frac{2}{r}\left\lfloor\frac{g}{r}\right\rfloor$.

Step 1 We show Cliff $(E)>\frac{2}{r}(g-1)-\frac{2}{r}\left\lfloor\frac{g}{r}\right\rfloor$ if $2 g+2<d \leq r(g-1)$.

Denote $t:=d-2(g-1)$. The first part of Theorem 1.1 implies that

$$
\begin{aligned}
& \operatorname{Cliff}(E)-\frac{2}{r}(g-1)+\frac{2}{r}\left\lfloor\frac{g}{r}\right\rfloor \\
& >\frac{t}{r}-\frac{2}{r}\left(r+\frac{\left(2+\frac{t}{g-1}\right)^{2}}{4 r} g+\frac{r}{g}\right)+2+\frac{2}{r}\left\lfloor\frac{g}{r}\right\rfloor=: Q(t) .
\end{aligned}
$$

Then $Q(t)$ is a quadratic function with respect to $t$ with negative leading coefficient. Thus it suffices to show that $Q(t=5)>0$ and $Q(t=(r-2)(g-1))>0$ which can be easily checked by direct computations.

Step 2 We show Cliff $(E) \geq \frac{2}{r}(g-1)-\frac{2}{r}\left\lfloor\frac{g}{r}\right\rfloor$ if $-2\left(\left\lfloor\frac{g}{r}\right\rfloor-r\right) \leq d-2(g-1) \leq 4$. 


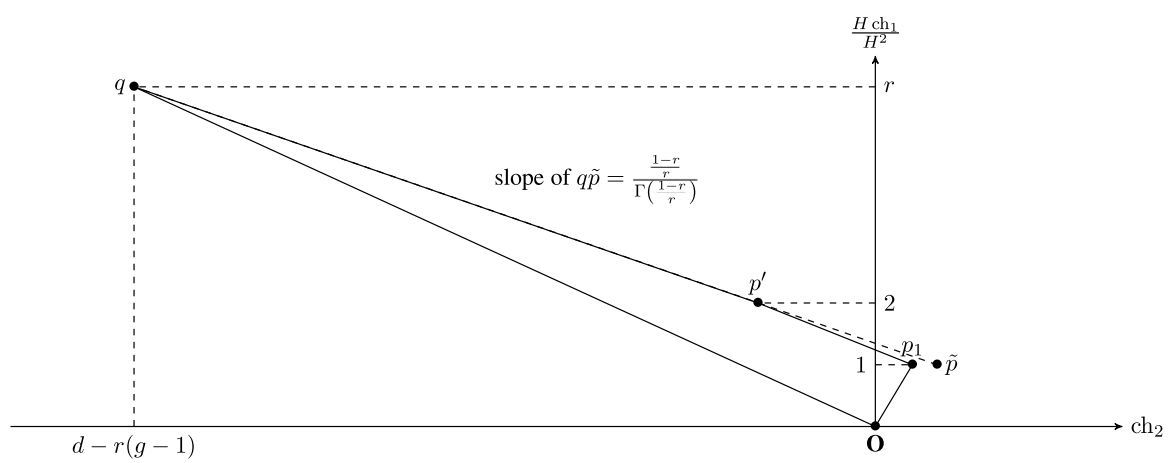

Fig. 6 The polygon $p_{\iota_{*} E}$ is inside the polygon $o p_{1} p^{\prime} q$

Applying Proposition 3.3 for the push-forward $\iota_{*} E$ implies that there exists $\epsilon>0$ such that its Harder-Narasimhan filtration with respect to $\sigma_{0, \alpha}$ for positive $\alpha<\epsilon$ is a fixed sequence

$$
0=\tilde{E}_{0} \subset \tilde{E}_{1} \subset \cdots \subset \tilde{E}_{n-1} \subset \tilde{E}_{n}=\iota_{*} E
$$

and

$$
\operatorname{Cliff}(E) \geq g+1-\frac{l(E)}{r}
$$

where $l(E):=\sum_{i=1}^{n}\left\lfloor\left\|p_{i} p_{i-1}\right\|\right\rfloor$ and $p_{i}=\bar{Z}\left(\tilde{E}_{i}\right)$. Thus it is suffices to show that

$$
l(E) \leq g(r-2)+2\left\lfloor\frac{g}{r}\right\rfloor+r+2 .
$$

Since $\tilde{E}_{1}$ is a sheaf supported in dimension $\geq 1$ and $\tilde{E}_{1} \in \mathcal{T}^{0}$, we get $\frac{H \operatorname{ch}_{1}\left(\tilde{E}_{1}\right)}{H^{2}}$ is a positive integer. We first treat with the case that $\frac{H \mathrm{ch}_{1}\left(\tilde{E}_{1}\right)}{H^{2}} \geq 2$. By Lemma 3.2, $\beta_{1} \geq-1+1 / r$. Applying the same argument as in Lemma 3.4 implies that the polygon $P_{l_{*} E}$ is contained in the triangle $o p^{\prime} q$ where the slope of $q p^{\prime}$ is $\frac{-1+1 / r}{\Gamma(-1+1 / r)}$ and the vertical coordinate of the point $p^{\prime}$ is equal to 2, see Fig. 6.

Denote by $\tilde{p}$ the point along the line $p^{\prime} q$ with the vertical coordinate equal to 1 . The coordinates of two points $p^{\prime}$ and $\tilde{p}$ are

$$
p^{\prime}=\left(d-2(g-1)-\frac{r-2}{r}(g+r), 2\right) \quad \text { and } \quad \tilde{p}=\left(d-2(g-1)+\frac{g}{r}-r, 1\right) .
$$

Note that the length $\|q \tilde{p}\|$ does not depend on $d$,

$$
\left\|p^{\prime} q\right\|=\frac{r-2}{r-1}\|\tilde{p} q\|=\frac{r-2}{r-1} \sqrt{\left((r-2)(g-1)+\frac{g}{r}-r\right)^{2}+4 g(r-1)^{2}}
$$




$$
<\frac{r-2}{r-1}\left((r-2)(g-1)+\frac{g}{r}+r+\frac{2 r}{g}\right)
$$

The horizontal coordinate of $p^{\prime}$ is negative and is bigger than $-g+r+2$. Thus if $r \geq 4$, we have

$$
\left\|o p^{\prime}\right\| \leq \sqrt{16 g+(g-r-2)^{2}} \leq g+r+\frac{1}{r}-\frac{6}{5} .
$$

This implies $l(E) \leq\left\lfloor\left\|o p^{\prime}\right\|+\left\|p^{\prime} q\right\|\right\rfloor \leq g(r-2)+2\left\lfloor\frac{g}{r}\right\rfloor+r+2$, so inequality (21) holds.

Now assume $\operatorname{ch}_{1}\left(\tilde{E}_{1}\right)=H$. By Lemma 3.2(b), we have $\beta_{2} \leq \frac{1}{r}$. Therefore $\operatorname{ch}_{2}\left(\tilde{E}_{1}\right) \leq\left\lfloor\frac{\Gamma\left(\beta_{2}\right)}{\beta_{2}}\right\rfloor=\left\lfloor\frac{g}{r}\right\rfloor-r$. We consider three different cases:

Case I $\left|\operatorname{ch}_{2}\left(\tilde{E}_{1}\right)\right| \leq\left\lfloor\frac{g}{r}\right\rfloor-r$

Case II $\lambda:=-\left\lfloor\frac{g}{r}\right\rfloor+r-\frac{g}{2 r} \leq \operatorname{ch}_{2}\left(\tilde{E}_{1}\right) \leq-\left\lfloor\frac{g}{r}\right\rfloor+r-1$

Case III $\operatorname{ch}_{2}\left(\tilde{E}_{1}\right) \leq \lambda$

We first assume $r \geq 5$, then the point $\tilde{\lambda}:=(\lambda, 1)$ lies on the right hand side of the line segment $o p^{\prime}$. In Case I, we have

$$
\left\|o p_{1}\right\| \leq \sqrt{4 g+\left(\left\lfloor\frac{g}{r}\right\rfloor-r\right)^{2}}<\left\lfloor\frac{g}{r}\right\rfloor+r+1,
$$

which implies $\left\lfloor\left\|o p_{1}\right\|\right\rfloor \leq\left\lfloor\frac{g}{r}\right\rfloor+r$. For Case II, write $s:=-\operatorname{ch}_{2}\left(\tilde{E}_{1}\right)-\left\lfloor\frac{g}{r}\right\rfloor+r$, then $1 \leq s \leq \frac{g}{2 r}$ and

$$
\left\|o p_{1}\right\|=\sqrt{4 g+\left(\left\lfloor\frac{g}{r}\right\rfloor+s-r\right)^{2}}<\left\lfloor\frac{g}{r}\right\rfloor+s+r .
$$

Thus $\left\lfloor\left\|o p_{1}\right\|\right\rfloor \leq\left\lfloor\frac{g}{r}\right\rfloor+s+r-1$. For $s=\frac{g}{2 r}$, we indeed have

$$
\|o \tilde{\lambda}\|=\sqrt{4 g+\left(\left\lfloor\frac{g}{r}\right\rfloor+\frac{g}{2 r}-r\right)^{2}}<\left\lfloor\frac{g}{r}\right\rfloor+\frac{g}{2 r}+r-1 .
$$

To provide an upper bound for the length $\left\|p_{1} p^{\prime}\right\|$, we define the function

$$
f(x):=\sqrt{4 g+\left(g \frac{r-2}{r}+\left\lfloor\frac{g}{r}\right\rfloor-x-2\right)^{2}} .
$$

If $0 \leq x \leq \frac{g}{2 r}$, one can easily show that

$$
f(x)<g \frac{r-2}{r}+\left\lfloor\frac{g}{r}\right\rfloor-x+\frac{2}{r-1}-\frac{r-2}{r-1} \frac{2 r}{g}+\delta,
$$


where $\delta=1$ if $x \in\left[0, \frac{g}{r^{2}}\right]$ and $\delta=2$ if $x \in\left(\frac{g}{r^{2}}, \frac{g}{2 r}\right]$.

In Case I, we know the point $p_{1}$ lies on the right hand side of $o p^{\prime}$, so the length of $p_{1} p^{\prime}$ is maximum when the horizontal coordinate of $p_{1}$ is maximum. But the horizontal coordinate of $p_{1}$ is less than or equal to $d-2(g-1)+\left\lfloor\frac{g}{r}\right\rfloor-r$ because $p_{1}$ lies on the left hand side of $\tilde{p}$, see Fig. 6, thus

$$
\left\|p_{1} p^{\prime}\right\| \leq f(0)
$$

In Case II, the length of $p_{1} p^{\prime}$ is maximum when the horizontal coordinate of $p^{\prime}$ is minimum, i.e. $d$ is minimum, hence

$$
\left\|p_{1} p^{\prime}\right\| \leq \sqrt{4 g+\left(-2\left(\left\lfloor\frac{g}{r}\right\rfloor-r\right)-\frac{r-2}{r}(g+r)-\operatorname{ch}_{2}\left(\tilde{E}_{1}\right)\right)^{2}}=f(s) .
$$

Here $s=-\operatorname{ch}_{2}\left(\tilde{E}_{1}\right)-\left\lfloor\frac{g}{r}\right\rfloor+r$ as before. Similarly, the length of $\tilde{\lambda} p^{\prime}$ is maximum when $d$ is minimum, so

$$
\left\|\tilde{\lambda} p^{\prime}\right\| \leq f\left(\frac{g}{2 r}\right)
$$

Now we apply the above upper bounds to prove inequality (21). In Case I, inequalities (23), (28) and (29) imply that

$$
\left\|p_{1} p^{\prime}\right\|+\left\|p^{\prime} q\right\|<g(r-2)+\left\lfloor\frac{g}{r}\right\rfloor+3
$$

Thus inequality (24) implies

$$
l(E) \leq\left\lfloor\left\|o p_{1}\right\|\right\rfloor+\left\lfloor\left\|p_{1} p^{\prime}\right\|+\left\|p^{\prime} q\right\|\right\rfloor \leq\left\lfloor\frac{g}{r}\right\rfloor+r+g(r-2)+\left\lfloor\frac{g}{r}\right\rfloor+2,
$$

so inequality (21) holds. Similarly, in Case II, inequalities (23), (28) and (30) imply that

$$
\left\|p_{1} p^{\prime}\right\|+\left\|p^{\prime} q\right\|<g(r-2)+\left\lfloor\frac{g}{r}\right\rfloor-s+4
$$

Therefore inequality (25) implies that

$$
\begin{aligned}
& l(E) \leq\left\lfloor\left\|o p_{1}\right\|\right\rfloor+\left\lfloor\left\|p_{1} p^{\prime}\right\|+\left\|p^{\prime} q\right\|\right\rfloor \\
& \quad \leq\left\lfloor\frac{g}{r}\right\rfloor+s+r-1+(r-2) g+\left\lfloor\frac{g}{r}\right\rfloor-s+3,
\end{aligned}
$$

thus again inequality (21) holds. Finally in Case III, we have

$$
\ell(E) \leq\left\lfloor\|o \tilde{\lambda}\|+\left\|\tilde{\lambda} p^{\prime}\right\|+\left\|p^{\prime} q\right\|\right\rfloor .
$$


Summing up inequalities (23), (26), (28) and (31) show that inequality (21) is satisfied.

Finally, we consider the case $r=4$. If $\left|\operatorname{ch}_{2}\left(\tilde{E}_{1}\right)\right| \leq\left\lfloor\frac{g}{4}\right\rfloor-4$, then $p_{1}$ lies to the right of $o p^{\prime}$ and the same argument as in the Case I above implies the claim. Otherwise, $\ell(E) \leq\left\lfloor\|o \tilde{\lambda}\|+\left\|\tilde{\lambda} p^{\prime}\right\|+\left\|p^{\prime} q\right\|\right\rfloor$ where $\tilde{\lambda}=(\lambda, 1)$ for $\lambda=-\left\lfloor\frac{g}{4}\right\rfloor+3$. Note that $\tilde{\lambda}$ lies to the right of $o p^{\prime}$. We know that the length of $\tilde{\lambda} p^{\prime}$ will be maximum when $d$ is minimum so

$$
\begin{aligned}
\|o \tilde{\lambda}\|+\left\|\tilde{\lambda} p^{\prime}\right\| & \leq \sqrt{4 g+\left(-\left\lfloor\frac{g}{4}\right\rfloor+3\right)^{2}}+\sqrt{4 g+\left(\left\lfloor\frac{g}{4}\right\rfloor+\frac{g}{2}-3\right)^{2}} \\
& <\left\lfloor\frac{g}{4}\right\rfloor+5+g \frac{1}{2}+\left\lfloor\frac{g}{4}\right\rfloor-1+\frac{2}{3}-\frac{16}{3 g}+1 .
\end{aligned}
$$

The second inequality follows from (28) for $x=1$. Summing up the above inequality with (23) proves our claim (21).

Step 3 We show $h^{0}(C, E)<2 r$ if $d<2(g-1)-2\left(\left\lfloor\frac{g}{r}\right\rfloor-r\right)$.

By using the same notations as in Step 2, we first consider the case $\operatorname{ch}_{1}\left(\tilde{E}_{1}\right) \neq H$. By Proposition 3.3, it suffices to show that

$$
k(d):=d+r(1-g)+\left\|o p^{\prime}\right\|+\left\|p^{\prime} q\right\|<4 r .
$$

One can easily check that the function

$$
k(d)=d+r(1-g)+\sqrt{16 g+\left(d-2(g-1)-\frac{r-2}{r}(g+r)\right)^{2}}+\left\|p^{\prime} q\right\|
$$

is increasing with respect to $d$, so

$$
k(d) \leq k\left(2(g-1)-2\left(\left\lfloor\frac{g}{r}\right\rfloor-r\right)-1\right)<4 r
$$

The last inequality comes from inequality (23) and some direct computations.

Thus we may assume $\operatorname{ch}_{1}\left(\tilde{E}_{1}\right)=H$. If $p_{1}$ is to the left of the line segment $o p^{\prime}$, then the total sum of $\left\|p_{i} p_{i-1}\right\|$ is also bounded by $\left\|o p^{\prime}\right\|+\left\|p^{\prime} q\right\|$. So we may always assume the polygon $o p_{1} p^{\prime} q$ is convex.

Define $t:=\tilde{p}_{(x)}-\operatorname{ch}_{2}\left(\tilde{E}_{1}\right)-\frac{g}{r}+\left\lfloor\frac{g}{r}\right\rfloor$, where $\tilde{p}_{(x)}=d-2(g-1)+g / r-r$ is the horizontal coordinate of the point $\tilde{p}$. We consider two different cases:

Case I when $0 \leq t<\frac{g}{2 r}$,

$$
\begin{aligned}
\left\|o p_{1}\right\| & =\sqrt{4 g+\left(-d+2(g-1)-\left\lfloor\frac{g}{r}\right\rfloor+t+r\right)^{2}} \\
& <-d+2(g-1)-\left\lfloor\frac{g}{r}\right\rfloor+t+3 r
\end{aligned}
$$


In particular, if $t \in\left[\frac{g}{r^{2}}, \frac{g}{2 r}\right)$, we can improve the bound by 1 ;

$$
\sqrt{4 g+\left(-d+2(g-1)-\left\lfloor\frac{g}{r}\right\rfloor+t+r\right)^{2}}<-d+2(g-1)-\left\lfloor\frac{g}{r}\right\rfloor+t+3 r-1
$$

Note that $\left\|p_{1} p^{\prime}\right\|=f(t)$ as that defined in (27).

Thus combining inequality (28) for $t \in\left[0, \frac{g}{2 r}\right]$ and inequality (23), we get

$$
l(E) \leq\left\lfloor\left\|o p_{1}\right\|\right\rfloor+\left\lfloor\left\|p_{1} p^{\prime}\right\|+\left\|p^{\prime} q\right\|\right\rfloor \leq 4 r+r(g-1)-d-1 .
$$

Hence the claim follows by Proposition 3.3.

Case II Suppose $\frac{g}{2 r} \leq t$. If $p_{1}$ lies on the left hand side of $o p^{\prime}$, the polygon $P_{l_{*} E}$ is inside the triangle $o p^{\prime} q$ and the claim follows from (32). Otherwise, the polygon $o p_{1} p^{\prime} q$ is convex and the summation of the length $\left\|o p_{1}\right\|+\left\|p_{1} p^{\prime}\right\|+\left\|p^{\prime} q\right\|$ is maximum when $t=\frac{g}{2 r}$.

Substituting $t=\frac{g}{2 r}$ into the formulas of $\left\|o p_{1}\right\|$ and $\left\|p_{1} p^{\prime}\right\|$, we have:

$$
\begin{aligned}
\left\|o p_{1}\right\| & =\sqrt{4 g+\left(-d+2(g-1)-\left\lfloor\frac{g}{r}\right\rfloor+\frac{g}{2 r}+r\right)^{2}} \\
& <-d+2(g-1)-\left\lfloor\frac{g}{r}\right\rfloor+\frac{g}{2 r}+3 r-2 . \\
\left\|p_{1} p^{\prime}\right\| & =f\left(\frac{g}{2 r}\right)<g \frac{r-2}{r}+\left\lfloor\frac{g}{r}\right\rfloor-\frac{g}{2 r}+\frac{2}{r-1}-\frac{r-2}{r-1} \frac{2 r}{g}+2, \text { by (28). }
\end{aligned}
$$

Together with (23) for $\left\|p^{\prime} q\right\|$, it follows that

$$
l(E) \leq\left\lfloor\left\|o p_{1}\right\|+\left\|p_{1} p^{\prime}\right\|+\left\|p^{\prime} q\right\|\right\rfloor \leq 4 r+r(g-1)-d-1,
$$

so the claim follows.

Proof of the second part of Theorem 1.1 for $r=3$. Let $E$ be a rank 3-semistable vector bundle on the curve $C$ of degree $d$. By Lemma 3.2, either $\beta_{1} \geq-\frac{1}{2}$ or $\operatorname{ch}\left(F_{2}\right)=$ $(3, H,-)$.

Case I If $\beta_{1} \geq-\frac{1}{2}$, since $\operatorname{ch}_{2}\left(\iota_{*} E\right) \leq 0$, the slope of the wall $\mathcal{W}$ for $\iota_{*} E$ is negative. Therefore, $\left|\beta_{2}\right|<\left|\beta_{1}\right| \leq \frac{1}{2}$. Lemma 3.4 implies that for each of the semistable factors $\tilde{E}_{i} / \tilde{E}_{i-1}$ in the Harder-Narasimhan filtration of $\iota_{*} E$ with respect to $\sigma_{0, \alpha}$ for positive $\alpha<\epsilon$, we have

$$
\left|\frac{H^{2} \operatorname{ch}_{2}\left(\tilde{E}_{i} / \tilde{E}_{i-1}\right)}{H \operatorname{ch}_{1}\left(\tilde{E}_{i} / \tilde{E}_{i-1}\right)}\right| \leq \frac{\Gamma(1 / 2)}{1 / 2} .
$$


Therefore $l(E) \leq\left\lfloor 3 \sqrt{4 g+(g / 2-2)^{2}}\right\rfloor=\lfloor 3(g / 2+2)\rfloor$. Note that $g \geq 3^{2}=9$, Proposition 3.3 implies that

$$
\operatorname{Cliff}(E) \geq g+1-\frac{l(E)}{3}=g-\frac{1}{3}\left\lfloor\frac{3 g}{2}\right\rfloor-1 \geq \frac{2}{3}(g-1)-\frac{2}{3}\left\lfloor\frac{g}{3}\right\rfloor .
$$

Case II If $\operatorname{ch}\left(F_{2}\right)=(3, H,-)$, then $\beta_{2} \leq \frac{\operatorname{ch}_{1}\left(F_{2} / T\left(F_{2}\right)\right) \cdot H}{3 H^{2}} \leq \frac{1}{3}$. When $d \geq 2(g-1)-$ $2\left(\left\lfloor\frac{g}{3}\right\rfloor-3\right)$, define $s:=-\operatorname{ch}_{2}\left(\tilde{E}_{1}\right)-\left\lfloor\frac{g}{3}\right\rfloor+3$, then using the same argument as in Step 2 for $r \geq 4$, if $\operatorname{ch}_{1}\left(\tilde{E}_{1}\right) \neq H$, then for $g \geq 9$ and $g \neq 11$, we have

$$
\begin{aligned}
l(E) & =\left\lfloor\left\|o p^{\prime}\right\|+\left\|p^{\prime} q\right\|\right\rfloor=\left\lfloor 2 \sqrt{\left(\frac{g}{3}-1\right)^{2}+g}+\sqrt{\left(d-\frac{7}{3} g+1\right)^{2}+16 g}\right\rfloor \\
& \leq\left\lfloor 2 \sqrt{\left(\frac{g}{3}-1\right)^{2}+g}+\sqrt{(g-5)^{2}+16 g}\right\rfloor \leq g+2\left\lfloor\frac{g}{3}\right\rfloor+5
\end{aligned}
$$

which shows inequality (21) holds for $r=3$. The only remaining case that the last inequality does not hold is when $g=11$, but the formula (35) is less than or equal to 22. Therefore, we may assume $\operatorname{ch}_{1}\left(\tilde{E}_{1}\right)=H$. Now the arguments in Step 2, Case I, II, and III in the proof of Theorem 1.1 for $r \geq 4$, are valid for $r=3$, thus $\mathrm{Cliff}_{3}(E) \geq \frac{2}{3}(g-1)-\frac{2}{3}\left\lfloor\frac{g}{3}\right\rfloor$.

When $d<2(g-1)-2\left(\left\lfloor\frac{g}{3}\right\rfloor-3\right)$, define $t=\tilde{p}_{(x)}-\operatorname{ch}_{2}\left(\tilde{E}_{1}\right)-\frac{g}{3}+\left\lfloor\frac{g}{3}\right\rfloor$, then again the computations in Step 3, Case I, II are valid for $r=3$, hence $h^{0}(F)<6$. Therefore, the second part of Theorem 1.1 for $r=3$ and $g \geq 9$ follows by Theorem 4.1.

Proof of the second part of Theorem 1.1 for $r=2$ Let $E$ be a semistable rank 2-vector bundle on the curve $C$. Assume there exists a wall $\mathcal{W}$ for $\iota_{*} E$ and $0 \rightarrow$ $F_{1} \rightarrow \iota_{*} E \rightarrow F_{2} \rightarrow 0$ is the destabilizing sequence as that in Lemma 3.1. As $H \operatorname{ch}_{1}\left(F_{1}\right), H \operatorname{ch}_{1}\left(F_{2}\right)>0$, we may assume $\operatorname{ch}_{1}\left(F_{1}\right)=\operatorname{ch}_{a}\left(F_{2}\right)=H$. By Lemma 3.2, we may assume $\operatorname{ch}\left(F_{1}\right)=(2, H, s)$ and $\operatorname{ch}\left(F_{2}\right)=(-2, H, 2(1-g)+$ $d-s)$. Since $H \operatorname{ch}_{1}\left(F_{i}\right) / H^{2}=1$ is minimal, both objects $F_{1}$ and $F_{2}$ are $\sigma_{0, \alpha}$-stable for any $\alpha>0$. Therefore, $F_{1}$ and $F_{2}$ are the Harder-Narasimhan factors of $\iota_{*} E$ with respect to $\sigma_{0, \alpha}$ where $0<\alpha \ll 1$. By [2, Theorem 2.15],

$$
-\chi\left(F_{2}, F_{2}\right)=H^{2}+4(2(1-g)+d-s)-8 \geq-2 \Longrightarrow s \leq d-\frac{3 g}{2} .
$$

Since $F_{1}$ destabilizes $\iota_{*} E$, we have

$$
\frac{s}{H^{2}}>\frac{\operatorname{ch}_{2}\left(\iota_{*} E\right)}{H \operatorname{ch}_{1}\left(\iota_{*} E\right)} \Longrightarrow s>\frac{d}{2}-g+1 .
$$


Combining the two inequalities, we get

$$
\frac{d}{2}-g+1<s \leq d-\frac{3 g}{2} \Longrightarrow d>g+2
$$

Note that $d \leq 2 g-2$, we have $s \leq \frac{g}{2}-2$. We also have $s>\frac{d}{2}-g+1>-\frac{g}{2}+2$. In particular, $|s| \leq \frac{g}{2}-2$. Proposition 3.3 implies that

$$
h^{0}(C, E) \leq-g+1+\frac{d}{2}+\frac{1}{2}\left\lfloor\sqrt{4 g+s^{2}}+\sqrt{4 g+(2(g-1)-d+s)^{2}}\right\rfloor
$$

Note that

$$
\begin{aligned}
& \sqrt{4 g+s^{2}}+\sqrt{4 g+(2(g-1)-d+s)^{2}} \\
& \leq \sqrt{4 g+\left(\frac{g}{2}-2\right)^{2}}+\sqrt{4 g+\left(2(g-1)-d+d-\frac{3 g}{2}\right)^{2}}=g+4
\end{aligned}
$$

As for the ' $\leq$ ', equality can hold only if $|s|=\frac{g}{2}-2$ and $s=d-\frac{3 g}{2}$. Since $d>g+2$, we must have $d=2(g-1)$ and $s=d-\frac{3 g}{2}$. Since $s$ is an integer, $g$ must be even in this case.

Hence when $g$ is odd, we have $\left\lfloor\sqrt{4 g+s^{2}}+\sqrt{4 g+(2(g-1)-d+s)^{2}}\right\rfloor \leq g+$ 3. Together with (36), we have $h^{0}(C, E) \leq-\left\lfloor\frac{g+1}{2}\right\rfloor+3+\frac{d}{2}$.

When $g$ is even, by (36) and (38), we directly have $h^{0}(C, E) \leq-\left\lfloor\frac{g+1}{2}\right\rfloor+3+\frac{d}{2}$ as well.

Hence when there exists a wall for $\iota_{*} E$, we have

$$
\operatorname{Cliff}(E) \geq \frac{d}{2}+\left\lfloor\frac{g+1}{2}\right\rfloor-3-\frac{d}{2}+2=g-1-\left\lfloor\frac{g}{2}\right\rfloor .
$$

Now assume there is no wall $\mathcal{W}$ for $\iota_{*} E$ and it is $\sigma_{0, \alpha}$-semistable where $\alpha \rightarrow 0$. Denote $x=d-2(g-1)$ and $p_{1}=\bar{Z}\left(\iota_{*} E\right)$, so $\left\|o p_{1}\right\|=\sqrt{x^{2}+16 g}$. Proposition 3.3 implies that

$$
\operatorname{Cliff}(E) \geq g+1-\frac{1}{2}\left\lfloor\sqrt{x^{2}+16 g}\right\rfloor
$$

Thus for $-g+4<x \leq 0$, we have $\operatorname{Cliff}(E) \geq g-1-\left\lfloor\frac{g}{2}\right\rfloor$. If $x \leq-g+4$, then again Proposition 3.3 gives

$2 h^{0}(C, E) \leq x+\sqrt{x^{2}+16 g}=\frac{16 g}{\sqrt{x^{2}+16 g}-x} \leq \frac{16 g}{\sqrt{(g-4)^{2}+16 g}+g-4}=8$.

Therefore the second part of Theorem 1.1 for $r=2$ follows by the fact that $\mathrm{Cliff}_{2}(C) \leq$ $\mathrm{Cliff}_{1}(C)=g-1-\left\lfloor\frac{g}{2}\right\rfloor$. 


\subsection{Higher Picard number case}

Theorem 1.1 still holds when the ample divisor $H$ satisfies Assumption (*). Assumption (*) $H^{2}$ divides $H \cdot D$ for all curve classes $D$ on $X$.

We explain how to adapt all our arguments from Picard rank one to this more general case.

Let $\Lambda_{H} \cong \mathbb{Z}^{3}$ denote the image of the map

$$
\mathbf{v}_{H}: K(X) \rightarrow \mathbb{R}^{3}, \quad E \mapsto\left(\operatorname{rk}(E), H \operatorname{ch}_{1}(E), \operatorname{ch}_{2}(E)\right)
$$

Consider stability conditions for which the central charge factors via $\mathbf{v}_{H}$, and denote the space of such stability conditions by $\operatorname{Stab}_{H}(X)$. The pair $\sigma_{\beta, \alpha}:=\left(\operatorname{Coh}^{\beta} X, Z_{\beta, \alpha}\right)$ defines a stability condition on $D^{b}(X)$ and there is a continuous map from $\Gamma_{+} \rightarrow$ $\operatorname{Stab}_{H}(X)$. The slope function $v_{\beta, \alpha}$ is defined in the same way. All the propositions in Sect. 2 hold for the higher Picard rank case. The Chern characters in part (a) in Lemma 3.2 should be modified to $H \cdot \operatorname{ch}\left(F_{1}\right)=H^{2}$. All the other statements do not rely on the Picard rank.

\section{Smooth plane curves}

Our method to control the dimension of global sections of semistable vector bundles (first part of Theorem 1.1) can be generalized to curves on more general surfaces, especially for Fano surfaces. As a case study, we follow the argument for curves on K3 surfaces to set up a bound for smooth projective plane curves and finally compute their Clifford indices. We first review Bridgeland stability conditions on the projective plane.

\subsection{Review: space of geometric stability conditions on $D^{b}\left(P^{2}\right)$}

The space of geometric stability conditions on the projective plane $\mathbf{P}^{2}$ is similar but slightly different with that of a K3 surface with Picard number one. In the projective plane case, the curve $\Gamma$ is replaced by the Le Potier curve (see $[6,7,12,20]$ ). Since the definition of Le Potier curve is rather involved, we will only use a simpler version $\tilde{\Gamma}$ which is enough for our purpose.

Definition 5.1 Let $\tilde{\gamma}: \mathbb{R} \rightarrow \mathbb{R}$ be a 1-periodic function. When $x \in\left[-\frac{1}{2}, \frac{1}{2}\right]$,

$$
\tilde{\gamma}(x):= \begin{cases}\frac{1}{2} x^{2}-\frac{3}{2}|x|+1 & \text { if } x \neq 0 \\ 0 & \text { if } x=0\end{cases}
$$

Let $\tilde{\Gamma}(x):=\frac{1}{2} x^{2}-\tilde{\gamma}(x)$. By abuse of notations, we also denote the graph of $\tilde{\Gamma}$ by the curve $\tilde{\Gamma}$. 
For $\beta \in \mathbb{R}$ and $\alpha>\tilde{\Gamma}(\beta)$, we define the central charge $Z_{\beta, \alpha}: K\left(\mathbf{P}^{2}\right) \rightarrow \mathbb{C}$ as

$$
Z_{\beta, \alpha}(E):=-\operatorname{ch}_{2}(E)+\alpha \operatorname{rk}(E)+i\left(\operatorname{ch}_{1}(E) . H-\beta \operatorname{rk}(E)\right) .
$$

By [12, Proposition 1.10], we get a slice of stability conditions $\sigma_{\beta, \alpha}=\left(\operatorname{Coh}^{\beta} \mathbf{P}^{2}, Z_{\beta, \alpha}\right)$ parameterized by $\tilde{\Gamma}_{+}$. Results of stability condition and wall-crossings (Theorem 2.2, Remark 2.3 and Proposition 2.4) all hold without any change. One should be cautious that the end points of the first wall may not be on the curve $\tilde{\Gamma}$.

\subsection{Upper bound on the dimension of global sections}

Let $C$ be a degree $l$ smooth irreducible curve in the projective plane $\mathbf{P}^{2}$. Denote $\iota: C \hookrightarrow \mathbf{P}^{2}$ the embedding morphism and $H:=\mathcal{O}_{\mathbf{P}^{2}}(1)$. We recollect lemmas from the case of K3 surfaces. The next lemma generalizes [8, Lemma 3.2] to objects in $D^{b}\left(\mathbf{P}^{2}\right)$.

Lemma 5.2 Fix an object $F \in \operatorname{Coh}^{0} \mathbf{P}^{2}$ which is $\sigma_{0, \alpha}$-semistable for any positive real number $\alpha \ll 1$ and $\operatorname{ch}_{1}(F) \neq 0$. Then

$$
\operatorname{hom}\left(\mathcal{O}_{\mathbf{P}^{2}}, F\right) \begin{cases}=\operatorname{rk}(F)+\frac{3}{2} H \operatorname{ch}_{1}(F)+\operatorname{ch}_{2}(F) & \text { when } \frac{\operatorname{ch}_{2}(F)}{H \cdot \operatorname{ch}_{1}(F)}>-\frac{3}{2} \\ \leq \operatorname{rk}(F)-\frac{\operatorname{ch}_{1}(F)^{2}}{2 \operatorname{ch}_{2}(F)} & \text { when } \operatorname{ch}_{2}(F)<0\end{cases}
$$

Proof We first assume $\frac{\operatorname{ch}_{2}(F)}{H \operatorname{ch}_{1}(F)}>-\frac{3}{2}$. The object $\mathcal{O}_{\mathbf{P}^{2}}(-3)[1] \in \operatorname{Coh}^{0} \mathbf{P}^{2}$ is $\sigma_{0, \alpha^{-}}$ semistable and $v_{0, \alpha}\left(\mathcal{O}_{\mathbf{P}^{2}}(-3)[1]\right)=-\frac{3}{2}<v_{0, \alpha}(F)$, thus $\operatorname{Hom}\left(F, \mathcal{O}_{\mathbf{P}^{2}}(-3)[i]\right)=0$, for $i \leq 1$. By Serre duality, we have $\operatorname{Hom}\left(\mathcal{O}_{\mathbf{P}^{2}}, F[2-i]\right)=0$ for $i \leq 1$. Since both $F$ and $\mathcal{O}_{\mathbf{P}^{2}}$ are in the heart $\operatorname{Coh}^{0} \mathbf{P}^{2}$, we have $\operatorname{Hom}\left(\mathcal{O}_{\mathbf{P}^{2}}, F[i]\right)=0$, for $i \leq-1$. Therefore,

$$
\operatorname{Hom}\left(\mathcal{O}_{\mathbf{P}^{2}}, F\right)=\chi\left(\mathcal{O}_{\mathbf{P}^{2}}, F\right)=\operatorname{rk}(F)+\frac{3}{2} H \operatorname{ch}_{1}(F)+\operatorname{ch}_{2}(F)
$$

Now assume $\operatorname{ch}_{2}(F)<0$. Define the object $K \in D^{b}\left(\mathbf{P}^{2}\right)$ as the canonical extension

$$
0 \rightarrow F \rightarrow K \rightarrow \mathcal{O}_{\mathbf{P}^{2}}[1] \otimes\left(\operatorname{Ext}^{1}\left(\mathcal{O}_{\mathbf{P}^{2}}[1], F\right) \rightarrow\right)^{*} 0
$$

in $\operatorname{Coh}^{\epsilon} \mathbf{P}^{2}$ for sufficiently small $\epsilon>0$. We have ch $(K)=\left(\operatorname{rk}(F)-h, \operatorname{ch}_{1}(F), \operatorname{ch}_{2}(F)\right)$, where $h$ denotes $\operatorname{dim} \operatorname{Ext}^{1}\left(\mathcal{O}_{\mathbf{P}^{2}}[1], F\right)=\operatorname{hom}\left(\mathcal{O}_{\mathbf{P}^{2}}, F\right)$. The object $K$ is semistable on the wall that the objects $F$ and $\mathcal{O}_{\mathbf{P}^{2}}[1]$ have the same phase, in particular, $\Delta(K) \geq 0$ :

$$
\begin{aligned}
0 & \leq\left(H \operatorname{ch}_{1}(K)\right)^{2}-2 \operatorname{ch}_{2}(K)(\operatorname{rk}(F)-h) \\
& \Longrightarrow h \leq \operatorname{rk}(F)-\frac{\left(H \operatorname{ch}_{1}(K)\right)^{2}}{2 \operatorname{ch}_{2}(K)}=\operatorname{rk}(F)-\frac{\left(H \operatorname{ch}_{1}(F)\right)^{2}}{2 \operatorname{ch}_{2}(F)} .
\end{aligned}
$$


Note that when $\frac{\operatorname{ch}_{2}(F)}{H \mathrm{ch}_{1}(F)} \in\left(-1,-\frac{1}{2}\right)$, we always have

$$
\operatorname{rk}(F)+\frac{3}{2} H \operatorname{ch}_{1}(F)+\operatorname{ch}_{2}(F)>\operatorname{rk}(F)-\frac{\operatorname{ch}_{1}(F)^{2}}{2 \operatorname{ch}_{2}(F)} .
$$

By the lemma, there is no $\sigma_{0, \alpha}$-semistable object with $\frac{\operatorname{ch}_{2}(F)}{H \operatorname{ch}_{1}(F)} \in\left(-1,-\frac{1}{2}\right)$.

Define the function $L:(a, b) \in \mathbb{H}=\mathbb{R} \times \mathbb{R}^{>0} \rightarrow \mathbb{R}^{>0}$ such that

$$
L(a, b)= \begin{cases}\frac{3}{2} b+a, & \text { if } \quad \frac{a}{b} \in I:=[-1,+\infty) \\ -\frac{b^{2}}{2 a}, & \text { if } \quad \frac{a}{b} \in J:=(-\infty,-1] .\end{cases}
$$

Note that $L(a, b)>0$ for any pair $(a, b) \in \mathbb{H}$.

Lemma 5.3 The function L satisfies the triangle inequality in $\mathbb{H}$, in other words, for any two vectors $v_{1}=\left(a_{1}, b_{1}\right)$ and $v_{2}=\left(a_{2}, b_{2}\right)$ in $\mathbb{H}$, we have $L\left(v_{1}+v_{2}\right) \leq L\left(v_{1}\right)+L\left(v_{2}\right)$. Moreover, $L(k v)=k L(v)$ for any $v \in \mathbb{H}$ and $k>0$.

Proof The second claim follows clearly by definition. To prove the first claim, we consider four different cases.

(a) If both $a_{1} / b_{1}$ and $a_{2} / b_{2}$ are in $I=[-1,+\infty)$, then $L\left(v_{1}+v_{2}\right)=L\left(v_{1}\right)+L\left(v_{2}\right)$.

(b) If both $a_{1} / b_{1}$ and $a_{2} / b_{2}$ are in $J=(-\infty,-1]$, then

$$
0 \leq \frac{-1}{a_{1}+a_{2}}\left(b_{1}^{2}\left(\frac{a_{2}}{a_{1}}\right)+b_{2}^{2}\left(\frac{a_{1}}{a_{2}}\right)-2 b_{1} b_{2}\right) .
$$

This implies

$$
\begin{aligned}
0 & \leq b_{1}^{2}\left(\frac{1}{a_{1}+a_{2}}-\frac{1}{a_{1}}\right)+b_{2}^{2}\left(\frac{1}{a_{1}+a_{2}}-\frac{1}{a_{2}}\right)+\frac{2 b_{1} b_{2}}{a_{1}+a_{2}} \\
& =-2 L\left(v_{1}+v_{2}\right)+2 L\left(v_{1}\right)+2 L\left(v_{2}\right) .
\end{aligned}
$$

(c) If $a_{1} / b_{1} \in I, a_{2} / b_{2} \in J$ and $\left(a_{1}+a_{2}\right) /\left(b_{1}+b_{2}\right) \in I$, then since $a_{2} / b_{2} \leq-1$, we have

$$
\frac{3}{2} b_{2}+a_{2} \leq-\frac{b_{2}^{2}}{2 a_{2}}
$$

which implies

$$
L\left(v_{1}+v_{2}\right)=\frac{3}{2}\left(b_{1}+b_{2}\right)+a_{1}+a_{2} \leq \frac{3}{2} b_{1}+a_{1}+-\frac{b_{2}^{2}}{2 a_{2}}=L\left(v_{1}\right)+L\left(v_{2}\right)
$$


(d) If $a_{1} / b_{1} \in I, a_{2} / b_{2} \in J$ and $\left(a_{1}+a_{2}\right) /\left(b_{1}+b_{2}\right) \in J$, then there is a non-negative real number $k<1$ such that $\left(a_{1}+k a_{2}\right) /\left(b_{1}+k b_{2}\right)=-1$, then case (c) implies that

$$
L\left(v_{1}+k v_{2}\right) \leq L\left(v_{1}\right)+k L\left(v_{2}\right)
$$

Therefore, case (b) gives

$$
L\left(v_{1}+v_{2}\right) \leq L\left((1-k) v_{2}\right)+L\left(v_{1}+k v_{2}\right) \leq(1-k) L\left(v_{2}\right)+k L\left(v_{2}\right)+L\left(v_{1}\right),
$$

which proves the claim.

Notation We will write $\overrightarrow{P Q}$ for the vector from $P$ to $Q$.

Fix a semistable rank $r$-vector bundle $E$ of degree $d$ on the curve $C$. The same argument as in [8, Proposition 3.4] implies that there exists $\epsilon>0$ such that the Harder-Narasimhan filtration of $\iota_{*} E$ is a fixed sequence

$$
0=\tilde{E}_{0} \subset \tilde{E}_{1} \subset \cdots \subset \tilde{E}_{n-1} \subset \tilde{E}_{n}=\iota_{*} E
$$

for all stability conditions $\sigma_{0, \alpha}$ where $0<\alpha<\epsilon$. Let $P_{\iota_{*} E}$ be the polygon with the extremal points $p_{i}:=\left(\operatorname{ch}_{2}\left(\tilde{E}_{i}\right), \operatorname{ch}_{1}\left(\tilde{E}_{i}\right)\right) \in \mathbb{R}^{2}$ for $i=0, \ldots, n$. Then Lemma 5.2 implies that

$$
h^{0}\left(X, \iota_{*} E\right) \leq \mathrm{rk}(E)+\sum_{i=1}^{n} L\left(\overrightarrow{p_{i} p_{i-1}}\right)
$$

Note that by definition, the curve with the equation $y=x^{2} / 2$ is above the curve $\tilde{\Gamma}$. Also when $0 \leq x<1$, the function $\tilde{\Gamma}(x) \leq-\frac{1}{2} x$. Therefore, any point $(\beta, \alpha)$ in the gray area in Fig. 7 gives a Bridgeland stability condition $\sigma_{\beta, \alpha}$.

Lemma 5.4 (Lemma 3.4) For any semistable factor $E_{i}:=\tilde{E}_{i} / \tilde{E}_{i-1}$ in the HarderNarasimhan filtration of $\iota_{*} E$, we have the slope $\frac{\operatorname{ch}_{2}\left(E_{i}\right)}{H \operatorname{ch}_{1}\left(E_{i}\right)} \in\left[\frac{d}{2 r l}-\frac{l}{2}, \frac{d}{2 r l}\right]$. When $d<r l$, the slope is either in the range $\left[\frac{d}{2 r l}-\frac{l}{2},-\frac{1}{2}\right]$ or $\left[-\frac{l-1}{2}, \frac{d}{r}-l+\frac{1}{2}\right]$.

Proof Let $0 \rightarrow F_{2} \rightarrow \iota_{*} E \rightarrow F_{1} \rightarrow 0$ be the destabilizing sequence at the wall $\mathcal{W}$ for $\iota_{*} E$ which passes a stability condition of form $\sigma_{0, \alpha}$. We have $\operatorname{ch}_{1}\left(\iota_{*} E\right)=r l H$ and $\operatorname{ch}_{1}\left(H^{0}\left(F_{1}\right)\right)=$ al $H$ for some integer $a \geq 0$. Denote $\operatorname{rk}\left(H^{-1}\left(F_{1}\right)\right)=\operatorname{rk}\left(F_{2}\right)=$ $s, \operatorname{ch}_{1}\left(H^{-1}\left(F_{1}\right)\right)=d_{1} H$ and $\operatorname{ch}_{1}\left(F_{2}\right)=d_{2} H$. Let $T\left(F_{2}\right)$ be the maximal torsion subsheaf of $F_{2}$, then $\operatorname{ch}_{1}\left(T\left(F_{2}\right)\right)=t l H$ for some integer $t \geq 0$. The same argument as in the first part of Lemma 3.1 implies that

$$
r l-a l \leq s l+t l .
$$

Therefore,

$$
\frac{H \operatorname{ch}_{1}\left(F_{2} / T\left(F_{2}\right)\right)}{s H^{2}}-\frac{H \operatorname{ch}_{1}\left(H^{-1}\left(F_{1}\right)\right)}{s H^{2}}=\frac{d_{2}-t l-d_{1}}{s}=\frac{r l-a l-t l}{s} \leq l .
$$


Fig. 7 First wall for $\iota_{*} E$

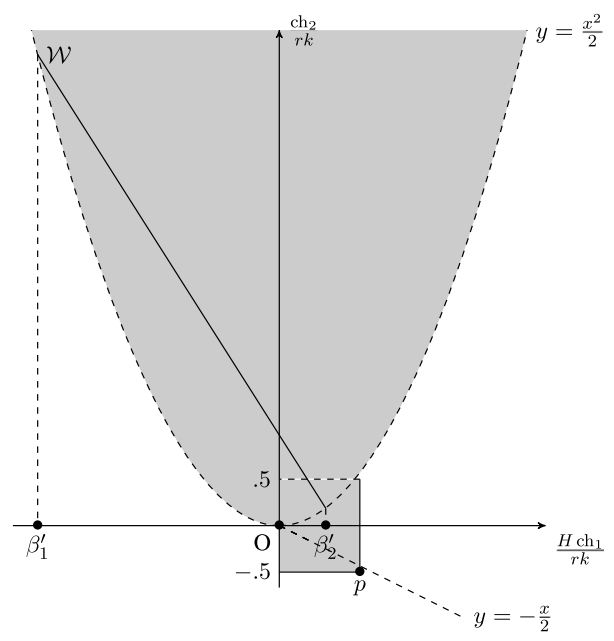

Now assume the wall $\mathcal{W}$ intersects the parabola with the equation $y=x^{2} / 2$ at two points $\left(\beta_{2}^{\prime}, \beta_{2}^{\prime 2} / 2\right)$ and $\left(\beta_{1}^{\prime}, \beta_{1}^{\prime 2} / 2\right)$ where $\beta_{1}^{\prime}<0<\beta_{2}^{\prime}$. By applying the same argument as in Lemma 3.1, the inequality (41) gives $\beta_{2}^{\prime}-\beta_{1}^{\prime} \leq l$. Proposition 2.4 implies that the slope of the wall $\mathcal{W}$ is

$$
\frac{\frac{1}{2}\left(\beta_{2}^{\prime}\right)^{2}-\frac{1}{2}\left(\beta_{1}^{\prime}\right)^{2}}{\beta_{2}^{\prime}-\beta_{1}^{\prime}}=\frac{\beta_{2}^{\prime}+\beta_{1}^{\prime}}{2}=\frac{\operatorname{ch}_{2}\left(\iota_{*} E\right)}{H \operatorname{ch}_{1}\left(\iota_{*} E\right)}=\frac{d-r \frac{l^{2}}{2}}{r l}=\frac{d}{r l}-\frac{l}{2} .
$$

Therefore, $\beta_{2}^{\prime} \leq \frac{d}{r l}$ and $\beta_{1}^{\prime} \geq \frac{d}{r l}-l$. By a similar argument as in Lemma 3.4, one can show that for each of the Harder-Narasimhan factors $E_{i}$,

$$
\frac{\beta_{1}^{\prime}}{2}=\frac{\beta_{1}^{\prime 2} / 2}{\beta_{1}^{\prime}} \leq \frac{\operatorname{ch}_{2}\left(E_{i}\right)}{H \operatorname{ch}_{1}\left(E_{i}\right)} \leq \frac{\beta_{2}^{\prime 2} / 2}{\beta_{2}^{\prime}}=\frac{\beta_{2}^{\prime}}{2}
$$

Thus the first claim follows.

Now assume $d<r l$, so $\beta_{2}^{\prime}<1$. If the wall $\mathcal{W}$ intersects the line with the equation $x=1$ at a point $(1, y)$ for $-1 / 2<y<1 / 2$, then the same argument as in Lemma 3.1 implies that

$$
1 \leq \frac{H \operatorname{ch}_{1}\left(F_{2} / T\left(F_{2}\right)\right)}{s H^{2}}
$$

and inequality (41) implies that

$$
1-l \leq \frac{H \operatorname{ch}_{1}\left(H^{-1}\left(F_{1}\right)\right)}{s H^{2}} \leq \beta_{1} .
$$

Therefore the wall $\mathcal{W}$ is below the line $L$ which has the same slope as $\mathcal{W}$ and passes through the point $\left(1-l, \frac{(l-1)^{2}}{2}\right)$. The line $L$ intersects the line $x=1$ at the point 
$\left(1, \frac{d}{r}-l+\frac{1}{2}\right)$. Thus the same argument as that in Lemma 3.4 shows that each slope $\frac{\mathrm{ch}_{2}\left(E_{i}\right)}{H \mathrm{ch}_{1}\left(E_{i}\right)}$ is in the range $\left[-\frac{1-l}{2}, \frac{d}{r}-l+\frac{1}{2}\right]$. If we have $y<-1 / 2$, then the wall intersects the line segment $\overline{o p}$ which has slope $-1 / 2$, see Fig. 7. Thus the same argument as in Lemma 3.4 implies that $\frac{\operatorname{ch}_{2}\left(E_{i}\right)}{H \operatorname{ch}_{1}\left(E_{i}\right)} \leq-\frac{1}{2}$ and the second claim follows.

Theorem 5.5 Let $C$ be a degree $l(\geq 5)$ smooth irreducible curve on the projective plane. Let $E$ be a semistable vector bundle with rank $r$ and degree $d$ such that $0 \leq$ $d \leq r l(l-3) / 2$. Then

$$
\operatorname{dim} H^{0}(C, E) \leq \begin{cases}r+\left(\frac{3}{2 l}+\frac{d}{2 r l^{2}}\right) d & \text { if } d \geq r l \\ \max \left\{3 r+d-r l, r+\frac{r l+r}{r l^{2}-d} d\right\} & \text { if } d<r l\end{cases}
$$

Proof When $d \geq r l$, Lemma 5.4 implies that the polygon $P_{l * E}$ is inside the triangle $o \tilde{p} q$ where $\tilde{p}=\left(\frac{d^{2}}{2 r l^{2}}, \frac{d}{l}\right)$ and $q=\left(-\frac{r l^{2}}{2}+d, r l\right)$. Then Lemma 5.3 and convexity of the polygon $P_{l * E}$ imply that

$$
\begin{aligned}
& h^{0}(C, E)=\operatorname{hom}\left(\mathcal{O}_{\mathbf{P}^{2}}, \iota_{*} E\right) \leq \sum_{i=1}^{n} L\left(\overrightarrow{p_{i} p_{i-1}}\right) \leq L(\overrightarrow{o \tilde{p}})+L(\overrightarrow{\tilde{p} q}) \\
& =\frac{3 d}{2 l}+\frac{d^{2}}{2 r l^{2}}+\frac{\left(r l-\frac{d}{l}\right)^{2}}{r l^{2}-2 d+\frac{d^{2}}{r l^{2}}} \\
& =\frac{3 d}{2 l}+\frac{d^{2}}{2 r l^{2}}+r \text {. }
\end{aligned}
$$

When $d<r l$, if the range of the slopes in Lemma 5.4 is given by $\left[\frac{d}{2 r l}-\frac{l}{2},-\frac{1}{2}\right]$, then we may let $\tilde{p}$ be at $\left(\frac{-r d l}{2 r l^{2}-2 r l-2 d}, \frac{r d l}{r l^{2}-r l-d}\right)$. Therefore,

$$
\begin{aligned}
h^{0}(C, E) & \leq L(\overrightarrow{o \tilde{p}})+L(\overrightarrow{\tilde{p} q})=\frac{r d l}{r l^{2}-r l-d}+\frac{\left(r l-\frac{r d l}{r l^{2}-r l-d}\right)^{2}}{r l^{2}-2 d-\frac{r d l}{r l^{2}-r l-d}} \\
& =\frac{r d l}{r l^{2}-r l-d}+\frac{r l}{r l^{2}-d}\left(r l-\frac{r d l}{r l^{2}-r l-d}\right) \\
& =r+d \frac{r l+r}{r l^{2}-d} .
\end{aligned}
$$

Also if the range of the slopes in Lemma 5.4 is given by $\left[-\frac{l-1}{2}, \frac{d}{r}-l+\frac{1}{2}\right]$, then we may let $\tilde{p}$ be at $\left(d-r l+\frac{r}{2}, r\right)$. Therefore,

$$
h^{0}(C, E) \leq L(\overrightarrow{o \tilde{p}})+L(\overrightarrow{\tilde{p} q})=\frac{3}{2} r+d-r l+\frac{r}{2}+\frac{r^{2}(l-1)^{2}}{r l^{2}-2 r l+r}=3 r+d-r l,
$$

which completes the proof. 
As an interesting consequence, part (i) of Mercat conjecture [24] holds for smooth plane curves.

Corollary 5.6 Let $C$ be a degree $l(\geq 5)$ smooth irreducible plane curve, then

$$
\operatorname{Cliff}_{r}(C)=l-4 \text {, }
$$

for any $r$.

Proof Let $E$ be a semistable vector bundle with rank $r$ and degree $l$, when $d \geq r l$, by Theorem 5.5

$$
\begin{aligned}
\operatorname{Cliff}(E) & \geq \frac{d}{r}-\frac{2}{r}\left(\frac{3}{2 l}+\frac{d}{2 r l^{2}}\right) d \geq \min _{d=r l, d=r l(l-3) / 2}\left\{\frac{d}{r}-\frac{2}{r}\left(\frac{3}{2 l}+\frac{d}{2 r l^{2}}\right) d\right\} \\
& =\min \left\{l-2 l\left(\frac{3}{2 l}+\frac{r l}{2 r l^{2}}\right), \frac{l(l-3)}{2}-\left(\frac{3}{2 l}+\frac{l-3}{4 l}\right) l(l-3)\right\} \\
& =\min \left\{l-4, \frac{l^{2}-6 l+9}{4}\right\}=l-4 .
\end{aligned}
$$

When $\frac{l^{2}-l}{l+1} r \leq d<r l$ and the upper bound for $H^{0}(C, E)$ is given by $3 r+d-r l$ in Theorem 5.5, then

$$
\operatorname{Cliff}_{r}(E) \geq \frac{d}{r}-\frac{2}{r}(2 r+d-r l)=l-4+l-\frac{d}{r}>l-4 .
$$

When $\frac{l^{2}-l}{l+1} r \leq d<r l$ and the upper bound for $H^{0}(C, E)$ is given by $r+\frac{r l+r}{r l^{2}-d} d$ in Theorem 5.5, then

$$
\operatorname{Cliff}_{r}(E) \geq \frac{d}{r}-\frac{2(l+1)}{r\left(l^{2}-l\right)} d=\frac{d}{r}\left(1-\frac{2 l+2}{l^{2}-l}\right) \geq \frac{l^{2}-l}{l+1}\left(1-\frac{2 l+2}{l^{2}-l}\right)>l-2-2 .
$$

When $d<\frac{l^{2}-l}{l+1} r$, by Theorem 5.5, $\operatorname{dim} H^{0}(C, E)<r+\frac{r l+r}{r l^{2}-r l} d<2 r$. On the other side, one may take $E=\mathcal{O}_{C}(1)^{\oplus r}$, then $\operatorname{Cliff}(E)=l-4$. Therefore, we have $\operatorname{Cliff}_{r}(C)=l-4$.

Acknowledgements The authors would like to thank Arend Bayer for many helpful conversations. We are grateful to Professor Peter Newstead for pointing out several related results and references. Both authors were supported by the ERC starting grant 'WallXBirGeom' 337039. C. L. is a Leverhulme Early Career Fellow and would like to acknowledge the Leverhulme Trust for the support. This project is also supported by the Royal Society URF $\backslash R 1 \backslash 201129$ 'Stability condition and application in algebraic geometry', and an EPSRC postdoctoral fellowship EP/T018658/1.

Open Access This article is licensed under a Creative Commons Attribution 4.0 International License, which permits use, sharing, adaptation, distribution and reproduction in any medium or format, as long as you give appropriate credit to the original author(s) and the source, provide a link to the Creative Commons licence, and indicate if changes were made. The images or other third party material in this article are included in the article's Creative Commons licence, unless indicated otherwise in a credit line to the material. If material is not included in the article's Creative Commons licence and your intended use is not permitted by statutory regulation or exceeds the permitted use, you will need to obtain permission directly from the copyright holder. To view a copy of this licence, visit http://creativecommons.org/licenses/by/4.0/. 


\section{References}

1. Bakker, B., Farkas, G.: The Mercat conjecture for stable rank 2 vector bundles on generic curves (2015). arXiv:1511.03253

2. Bayer, A., Macrì, E.: MMP for moduli of sheaves on K3s via wall-crossing: nef and movable cones, Lagrangian fibrations. Invent. Math. 198(3), 505-590 (2014). arXiv:1301.6968

3. Brambila-Paz, L., Grzegorczyk, I., Newstead, P.E.: Geography of Brill-Noether loci for small slopes. J. Algebr. Geom. 6(4), 645-669 (1995)

4. Bridgeland, T.: Stability conditions on triangulated categories. Ann. Math. (2) 166(2), 317-345 (2007). arXiv:math/0212237

5. Bridgeland, T.: Stability conditions on $K 3$ surfaces. Duke Math. J. 141(2), 241-291 (2008). arXiv:math/0307164

6. Coskun, I., Huizenga, J., Woolf, M.: The effective cone of the moduli space of sheaves on the plane (2014). arXiv:1401.1613

7. Drezet, J.-M., Le Potier, J.: Fibrés stables et fibrés exceptionnels sur $\mathbf{P}_{2}$. Ann. Sci. École Norm. Sup. (4) 18(2), 193-243 (1985)

8. Feyzbakhsh, S.: Mukai's program (reconstructing a K3 surface from a curve) via wall-crossing (2017). arxiv: 1710.06692

9. Feyzbakhsh, S.: An effective restriction theorem via wall-crossing and Mercat's conjecture (2021). arXiv: $1608.07825 \mathrm{v} 2$

10. Green, M., Lazarsfeld, R.: Special divisors on curves on a k3 surface. Invent. Math. 89, 357-370 (1987)

11. Happel, D., Reiten, I., Smalø, S.O.: Tilting in abelian categories and quasitilted algebras. Mem. Am. Math. Soc. 120(575), viii+88 (1996)

12. Li, C.: The space of stability conditions on the projective plane. Sel. Math. (N.S.) 23(4), 2927-2945 (2017)

13. Li, C.: On stability conditions for the quintic threefold. Invent. Math. 218(1), 301-340 (2019)

14. Lange, H., Mercat, V., Newstead, P.E.: On an example of Mukai. Glasg. Math. J. 54(2), 261-271 (2012)

15. Lange, H., Newstead, P.E.: Clifford indices for vector bundles on curves. In Affine Flag Manifolds and Principal Bundles, Trends Math., pp. 165-202. Birkhäuser/Springer Basel AG, Basel (2010)

16. Lange, H., Newstead, P.E.: On bundles of rank 3 computing Clifford indices. Kyoto J. Math. 53(1), 25-54 (2013)

17. Lange, H., Newstead, P.E.: Higher rank BN-theory for curves of genus 4. Rev. Mat. Complut. 45(9), 3948-3966 (2015)

18. Lange, H., Newstead, P.E.: Higher rank BN-theory for curves of genus 5. Rev. Mat. Complut. 29(3), 691-717 (2016)

19. Lange, H., Newstead, P.E.: Higher rank BN-theory for curves of genus 6. Int. J. Math. 29(2), 1850014 (2018)

20. Li, C., Zhao, X.: Birational models of moduli spaces of coherent sheaves on the projective plane (2016). arXiv:1603.05035

21. Maciocia, A.: Computing the walls associated to Bridgeland stability conditions on projective surfaces. Asian J. Math. 18(2), 263-279 (2014)

22. Mercat, V.: Le problème de Brill-Noether pour des fibrés stables de petite pente. J. Reine Angew. Math. 506, 1-41 (1999)

23. Mercat, V.: Fibrés stables de pente 2. Bull. Lond. Math. Soc. 33(5), 535-542 (2001)

24. Mercat, V.: Clifford's theorem and higher rank vector bundles. Int. J. Math. 13(07), 785-796 (2002)

Publisher's Note Springer Nature remains neutral with regard to jurisdictional claims in published maps and institutional affiliations. 